\title{
TRIGONOMETRIC WAVELETS FOR HERMITE INTERPOLATION
}

\author{
EWALD QUAK
}

\begin{abstract}
The aim of this paper is to investigate a multiresolution analysis of nested subspaces of trigonometric polynomials. The pair of scaling functions which span the sample spaces are fundamental functions for Hermite interpolation on a dyadic partition of nodes on the interval $[0,2 \pi)$. Two wavelet functions that generate the corresponding orthogonal complementary subspaces are constructed so as to possess the same fundamental interpolatory properties as the scaling functions. Together with the corresponding dual functions, these interpolatory properties of the scaling functions and wavelets are used to formulate the specific decomposition and reconstruction sequences. Consequently, this trigonometric multiresolution analysis allows a completely explicit algorithmic treatment.
\end{abstract}

\section{INTRODUCTION}

Trigonometric polynomials - being the simplest periodic analytic functions have recently become the object of investigations from the point of view of wavelet theory. For the basic terminology and fundamental concepts of wavelets, the reader is referred to the monograph of C. K. Chui [3]. A multiresolution analysis for $2 \pi$-periodic square-integrable functions consisting of finite-dimensional nested spaces of trigonometric polynomials was first studied in a paper by C. K. Chui and H. N. Mhaskar [4]. Their scaling functions and wavelets, however, do not possess interpolatory properties. Alternatively, a trigonometric multiresolution analysis can be based on fundamental functions of Lagrange interpolation. Trigonometric interpolants have a long history in approximation theory (see [14] and [17, Chapter 10]). Recently, A. A. Privalov [14] used specific interpolants to tackle the problem of finding orthogonal trigonometric polynomial bases of minimal degree for the space of $2 \pi$-periodic continuous functions. His results were then improved by D. Offin and K. Oskolkov [10], who used a periodized wavelet basis, and the final answer was given by R. A. Lorentz and A. A. Sahakian [9], who adopted a wavelet packet approach. Using Privalov's interpolants from [14], J. Prestin and E. Quak [11] explicitly computed the basis transformations connecting the spaces of trigonometric scaling functions and wavelets. The corresponding transformation matrices have circulant structure, thus giving rise to efficient decomposition and reconstruction algorithms that can be implemented using Fast Fourier Transform techniques. Prestin

Received by the editor May 4, 1994 and, in revised form, October 23, 1994.

1991 Mathematics Subject Classification. Primary 42A15, 41A05, 65D05.

Key words and phrases. Trigonometric wavelets, Hermite interpolation, decomposition and reconstruction sequences and algorithms. 
and Quak also formulated the duality principle for this trigonometric multiresolution analysis [12] and considered the decay rates for the trigonometric interpolatory scaling functions and wavelets [13].

Recently, Y. W. Koh, S. L. Lee and H. H. Tan [8] presented a general approach to non-stationary multiresolution analysis of the space of square-integrable $2 \pi$-periodic functions, which contains the trigonometric wavelets of Chui and Mhaskar [4] as a special case. It remains to be investigated how the trigonometric interpolatory wavelets of [11-14] fit into their framework and how their approach may be adapted to the use of two (or more) different types of scaling functions and wavelets in the respective sample and wavelet spaces. This paper describes one particular situation where two different scaling functions arise quite naturally, namely in trigonometric interpolation of Hermite data.

Other Hermite-type functions, which are not trigonometric polynomials, have already been studied in a wavelet context. For instance, P. Auscher [1] considers wavelets with boundary conditions on an interval based on cardinal Hermite Bspline functions, and T. N. T. Goodman [7] proves the existence of interpolatory Hermite spline wavelets on the real axis based on the B-spline theory of Schoenberg and Sharma. Trigonometric interpolants, however, as in the Lagrange case, enable a completely explicit description of the corresponding decomposition and reconstruction coefficients by means of circulant matrices.

In $\S 2$ of this paper, two different types of trigonometric scaling functions are constructed: one type whose function values in dyadic points are the fundamental Kronecker data and whose first derivatives in these points are all zero, and the other type for which the function values are all zero and the first derivatives are given by the Kronecker data. Certainly, these interpolants are well known, but for the sake of completeness and for later use, basic notations and properties are reviewed. The sample spaces spanned by these interpolants are identified as the same ones that were used by Chui and Mhaskar [4], but now the two different types of interpolatory scaling functions give rise to a Hermite interpolation operator instead of the quasiinterpolation operator defined in [4].

In $\S 3$, the trigonometric wavelets are constructed, which span the relative orthogonal complements for the sample spaces. These wavelets show the same interpolatory Hermite properties as the scaling functions and thus constitute high-frequency interpolants of the fundamental data, whereas the scaling functions on the same level can be considered as low-frequency interpolants. This is maybe the most interesting result in this context, as the wavelets for the Lagrange interpolants in [11] do not have this property - they interpolate the fundamental Lagrange data in the midpoints of the original knots.

The interpolation properties help to derive the two-scale (or reconstruction) relations for the Hermite multiresolution analysis in $\S 4$. A concise matrix representation using circulants is also given at this point. As usual in this context, the decomposition relations need more effort. First, in $\S 5$, the inner product matrix of the scaling functions is explicitly computed as well as the entries of its inverse, which are the coefficients of the biorthogonal bases of dual functions. The usefulness of these dual functions - as described in [3] and [5] for functions on the real axis - can be seen in $\S 6$, where they are used to establish the more complicated decomposition relations. Finally, $\S 7$ provides a short numerical example illustrating practical results and offers a discussion of open questions. The Appendix was added to provide some 
background material concerning an error estimate (similar to the one in [4]) for the Hermite interpolation operator of $\S 2$.

\section{Interpolatory HERMite-TYPe SCALING FUNCTIONS}

For $\ell \in \mathbb{N}$, the Dirichlet kernel $D_{\ell} \in T_{\ell}$ and the conjugate Dirichlet kernel $\tilde{D}_{\ell} \in T_{\ell}$ are defined as

$$
D_{\ell}(x)=\frac{1}{2}+\sum_{k=1}^{\ell} \cos k x= \begin{cases}\frac{\sin \left(\ell+\frac{1}{2}\right) x}{2 \sin \frac{x}{2}} & \text { for } x \notin 2 \pi \mathbb{Z}, \\ \ell+\frac{1}{2} & \text { for } x \in 2 \pi \mathbb{Z}\end{cases}
$$

and

$$
\tilde{D}_{\ell}(x)=\sum_{k=1}^{\ell} \sin k x=\left\{\begin{array}{lr}
\frac{\cos \frac{x}{2}-\cos \left(\ell+\frac{1}{2}\right) x}{2 \sin \frac{x}{2}} & \text { for } x \notin 2 \pi \mathbb{Z}, \\
0 & \text { for } x \in 2 \pi \mathbb{Z},
\end{array}\right.
$$

where $T_{\ell}$ denotes the linear space of trigonometric polynomials of degree $\ell$.

Following Zygmund [17, Vol. I, p.49], we recall that these kernels allow representation formulae for Fourier sums. In fact, let us start with the $\ell$ th partial Fourier sum

$$
S_{\ell}(f)(x)=\frac{a_{0}}{2}+\sum_{k=1}^{\ell}\left(a_{k} \cos k x+b_{k} \sin k x\right),
$$

where

$$
a_{k}=\frac{1}{\pi} \int_{0}^{2 \pi} f(t) \cos k t d t \quad \text { and } \quad b_{k}=\frac{1}{\pi} \int_{0}^{2 \pi} f(t) \sin k t d t
$$

are the usual Fourier coefficients of a function $f \in L_{2 \pi}^{2}$, i.e., a square-integrable $2 \pi$-periodic function. Then the conjugate $\ell$ th partial sum is defined as

$$
\tilde{S}_{\ell}(f)(x)=\sum_{k=1}^{\ell}\left(a_{k} \sin k x-b_{k} \cos k x\right),
$$

and it follows that for any $x \in[0,2 \pi]$

$$
S_{\ell}(f)(x)=\frac{1}{\pi} \int_{0}^{2 \pi} f(t) D_{\ell}(t-x) d t=2\left\langle f(\cdot), D_{\ell}(\cdot-x)\right\rangle
$$

and

$$
\tilde{S}_{\ell}(f)(x)=-\frac{1}{\pi} \int_{0}^{2 \pi} f(t) \tilde{D}_{\ell}(t-x) d t=-2\left\langle f(\cdot), \tilde{D}_{\ell}(\cdot-x)\right\rangle .
$$

Here and throughout, the inner product $\langle\cdot, \cdot\rangle$ of two functions $f$ and $g$ in $L_{2 \pi}^{2}$ is defined, as usual, by $\langle f, g\rangle=\frac{1}{2 \pi} \int_{0}^{2 \pi} f(x) \overline{g(x)} d x$.

The nodes for the interpolation processes of this section are equally spaced on the interval $[0,2 \pi)$ with a dyadic step size, i.e.,

$$
x_{j, n}=\frac{n \pi}{2^{j}} \quad \text { for any } j \in \mathbb{N}_{0} \text { and } n=0, \ldots, 2^{j+1}-1 .
$$

In the following, the relations between the nodes on consecutive levels such as $x_{j, n}=x_{j+1,2 n}$ or 'the midpoint of $x_{j, n}$ and $x_{j, n+1}$ is $x_{j+1,2 n+1}$ ' will be exploited frequently. 
Definition 2.1 (Scaling functions). For any $j \in \mathbb{N}_{0}$, consider two different kinds of kernels,

$$
\phi_{j, 0}^{0}(x):=\frac{1}{2^{2 j+1}} \sum_{\ell=0}^{2^{j+1}-1} D_{\ell}(x)
$$

and

$$
\phi_{j, 0}^{1}(x):=\frac{1}{2^{2 j+1}}\left(\tilde{D}_{2^{j+1}-1}(x)+\frac{1}{2} \sin \left(2^{j+1} x\right)\right)
$$

For $n=0, \ldots, 2^{j+1}-1$, define $\phi_{j, n}^{0}(x):=\phi_{j, 0}^{0}\left(x-x_{j, n}\right)$ and, in a similar way, $\phi_{j, n}^{1}(x):=\phi_{j, 0}^{1}\left(x-x_{j, n}\right)$. Furthermore, for notational convenience, let $\phi_{j, n}^{0}=$ $\phi_{j, n \bmod 2^{j+1}}^{0}$ and $\phi_{j, n}^{1}=\phi_{j, n \bmod 2^{j+1}}^{1}$ for any $n \in \mathbb{Z}$.

These functions are well known and have been studied in detail, e.g., in [17]. Closed formulae for them are given in

Lemma 2.1. For any $j \in \mathbb{N}_{0}$, we have

$$
\begin{aligned}
& \phi_{j, 0}^{0}(x)= \begin{cases}\frac{1}{2^{2 j+2}} \frac{\sin ^{2}\left(2^{j} x\right)}{\sin ^{2}\left(\frac{x}{2}\right)} & \text { for } x \notin 2 \pi \mathbb{Z}, \\
1 & \text { for } x \in 2 \pi \mathbb{Z},\end{cases} \\
& \phi_{j, 0}^{1}(x)= \begin{cases}\frac{1}{2^{2 j+2}}\left(1-\cos \left(2^{j+1} x\right)\right) \cot \frac{x}{2} & \text { for } x \notin 2 \pi \mathbb{Z}, \\
0 & \text { for } x \in 2 \pi \mathbb{Z},\end{cases}
\end{aligned}
$$

and their derivatives are given by

$$
\phi_{j, 0}^{0}{ }^{\prime}(x)= \begin{cases}\frac{1}{2^{j+2}} \frac{\sin \left(2^{j+1} x\right)}{\sin ^{2}\left(\frac{x}{2}\right)}-\frac{1}{2^{2 j+2}} \frac{\sin ^{2}\left(2^{j} x\right) \cot \left(\frac{x}{2}\right)}{\sin \left(\frac{x}{2}\right)} & \text { for } x \notin 2 \pi \mathbb{Z}, \\ 0 & \text { for } x \in 2 \pi \mathbb{Z}\end{cases}
$$

and

$$
\phi_{j, 0}^{1}{ }^{\prime}(x)= \begin{cases}\frac{1}{2^{2 j+3}} \frac{\cos \left(2^{j+1} x\right)-1}{\sin ^{2}\left(\frac{x}{2}\right)}+\frac{1}{2^{j+1}} \sin \left(2^{j+1} x\right) \cot \left(\frac{x}{2}\right) & \text { for } x \notin 2 \pi \mathbb{Z} \\ 1 & \text { for } x \in 2 \pi \mathbb{Z}\end{cases}
$$

Proof. The function $\phi_{j, 0}^{0}$ is actually the (positive) Fejér kernel and its representation (2.7) is listed in [17, Vol. I, p. 88, (3.1), (3.2)]. The representation (2.8) is given in [17, Vol. II, p.23, (6.8)]. The expressions (2.9) and (2.10) are then obtained by just taking derivatives. For the cases $x \in 2 \pi \mathbb{Z}$, the summation formulae (2.1) and (2.2) and their respective derivatives were used.

By evaluating the explicit formulae of Lemma 2.1, the following interpolatory properties can be established, taking into account that $\phi_{j, n}^{i}(x)=\phi_{j, 0}^{i}\left(x-x_{j, n}\right)$, for $i=0,1$, and that consequently only the interpolatory properties of $\phi_{j, 0}^{i}$, i.e., for the case $n=0$, need to be checked.

Theorem 2.1 (Interpolatory properties of the scaling functions). The following interpolatory properties hold for each $k, n=0, \ldots, 2^{j+1}-1$ :

$$
\phi_{j, n}^{0}\left(x_{j, k}\right)=\delta_{k, n} \quad \text { and } \quad \phi_{j, n}^{0}{ }^{\prime}\left(x_{j, k}\right)=0,
$$


and

$$
\phi_{j, n}^{1}\left(x_{j, k}\right)=0 \quad \text { and } \quad \phi_{j, n}^{1}{ }^{\prime}\left(x_{j, k}\right)=\delta_{k, n} .
$$

The values at the interlacing points $x_{j+1,2 k+1}, k=0, \ldots, 2^{j+1}-1$, are given by

$$
\begin{aligned}
& \phi_{j, n}^{0}\left(x_{j+1,2 k+1}\right)=\frac{1}{2^{2 j+2}} \sin ^{-2}\left(x_{j+2,2 k+1-2 n}\right), \\
& \phi_{j, n}^{0}{ }^{\prime}\left(x_{j+1,2 k+1}\right)=-\frac{1}{2^{2 j+2}} \frac{\cot \left(x_{j+2,2 k+1-2 n}\right)}{\sin ^{2}\left(x_{j+2,2 k+1-2 n}\right)},
\end{aligned}
$$

while

and

$$
\phi_{j, n}^{1}\left(x_{j+1,2 k+1}\right)=\frac{1}{2^{2 j+1}} \cot \left(x_{j+2,2 k+1-2 n}\right)
$$

$$
\phi_{j, n}^{1}{ }^{\prime}\left(x_{j+1,2 k+1}\right)=-\frac{1}{2^{2 j+2}} \sin ^{-2}\left(x_{j+2,2 k+1-2 n}\right) .
$$

Now, the sample spaces $V_{j}$ spanned by the translates $\phi_{j, n}^{0}$ and $\phi_{j, n}^{1}$ are introduced, which will be shown to form a trigonometric multiresolution analysis of $L_{2 \pi}^{2}$.

Definition 2.2. For $j \in \mathbb{N}_{0}$, the spaces $V_{j}$ are defined by

$$
V_{j}:=\operatorname{span}\left\{\phi_{j, n}^{0}, \phi_{j, n}^{1}: n=0, \ldots, 2^{j+1}-1\right\} .
$$

As a first step of studying the spaces $V_{j}$, the following result identifies the trigonometric polynomials which form alternative bases of these spaces.

Theorem 2.2. For any $j \in \mathbb{N}_{0}$, we have

$$
V_{j}=\operatorname{span}\left\{1, \cos x, \ldots, \cos \left(2^{j+1}-1\right) x, \sin x, \ldots, \sin 2^{j+1} x\right\} .
$$

Consequently,

$$
\operatorname{dim} V_{j}=2^{j+2}
$$

Proof. By the definition of the function $\phi_{j, 0}^{0}$, it is clear that $\phi_{j, 0}^{0}$ and its translates by $\frac{n \pi}{2^{j}}$ are elements of $T_{2^{j+1}-1} \subset \operatorname{span}\left\{1, \cos x, \ldots, \cos \left(2^{j+1}-1\right) x, \sin x, \ldots, \sin 2^{j+1} x\right\}$. Also, it is apparent that $\phi_{j, 0}^{1}$ is an element of this span. A translation of $\phi_{j, 0}^{1}$ by $\frac{n \pi}{2^{j}}$ does not cause any problem as the term $\sin 2^{j+1} x$ is not affected. Altogether, this means that $V_{j} \subset \operatorname{span}\left\{1, \cos x, \ldots, \cos \left(2^{j+1}-1\right) x, \sin x, \ldots, \sin 2^{j+1} x\right\}$. The equality follows from the interpolation conditions (2.11) and (2.12), which show that the functions spanning $V_{j}$ are indeed linearly independent, and therefore we have $\operatorname{dim} V_{j}=2^{j+2}$.

Moreover, Theorem 2.2 implies that

$$
V_{j} \subset V_{j+1}
$$


i.e., the spaces $V_{j}$ form a sequence of nested subspaces of $L_{2 \pi}^{2}$, the space of $2 \pi$ periodic square-integrable functions. With the notation $V_{-1}=\{0\}$, it is also clear that

$$
L_{2 \pi}^{2}=\cos _{L^{2}}\left(\bigcup_{j=-1}^{\infty} V_{j}\right) \text { and } \bigcap_{j=-1}^{\infty} V_{j}=\{0\} .
$$

A relation between $\phi_{j, 0}^{0}$ and $\phi_{j+1,0}^{0}$ as well as between $\phi_{j, 0}^{1}$ and $\phi_{j+1,0}^{1}$ based solely on dilation is incompatible with the periodicity of the functions involved. Using properties (2.7) and (2.8) of the scaling functions, we can compute that

$$
\phi_{j+1,0}^{0}(x)=\phi_{j, 0}^{0}(2 x) \frac{1}{4} \frac{\sin ^{2}(x)}{\sin ^{2}(x / 2)} \quad \text { and } \quad \phi_{j+1,0}^{1}(x)=\phi_{j, 0}^{1}(2 x) \frac{1}{4} \frac{\cot (x / 2)}{\cot (x)} .
$$

Note that the corrective factors $\frac{1}{4} \frac{\sin ^{2}(x)}{\sin ^{2}(x / 2)}$ and $\frac{1}{4} \frac{\cot (x / 2)}{\cot (x)}$, respectively, are independent of the level $j$.

Now, a Hermite-type interpolation operator can be introduced.

Definition 2.3. For any $j \in \mathbb{N}_{0}$, the interpolation operator $L_{j}$ mapping any realvalued differentiable $2 \pi$-periodic function $f$ into the space $V_{j}$ is defined as

$$
L_{j} f(x)=\sum_{n=0}^{2^{j+1}-1} f\left(x_{j, n}\right) \phi_{j, n}^{0}(x)+\sum_{n=0}^{2^{j+1}-1} f^{\prime}\left(x_{j, n}\right) \phi_{j, n}^{1}(x) .
$$

The following properties of the operators $L_{j}$ are therefore obvious:

$$
\begin{aligned}
& L_{j} f \in T_{2^{j+1}}, \\
& L_{j} f\left(x_{j, k}\right)=f\left(x_{j, k}\right) \text { and }\left(L_{j} f\right)^{\prime}\left(x_{j, k}\right)=f^{\prime}\left(x_{j, k}\right), k \in \mathbb{Z}, \\
& L_{j} f=f \text { for all } f \in V_{j} .
\end{aligned}
$$

It is possible to establish an error estimate for the Hermite interpolation operator $L_{j}$. For this purpose, define the space $W_{2}^{p}$ as the set of all functions $f \in L_{2 \pi}^{2}$ for which the second derivative $f^{\prime \prime}$ is $L^{p}$-integrable, where the $L^{p}$-norm of a $2 \pi$-periodic function $g$ is defined as

$$
\|g\|_{p}:=\left(\frac{1}{2 \pi} \int_{0}^{2 \pi}|g(x)|^{p} d x\right)^{1 / p}, \quad 1 \leq p \leq \infty,
$$

with the usual supremum modification for $p=\infty$.

Theorem 2.3. For a function $f \in W_{2}^{p}, 1 \leq p \leq \infty$, the following error estimate for the Hermite interpolation operator $L_{j}$ holds:

$$
\left\|f-L_{j} f\right\|_{p} \leq C_{p, j} 2^{-2 j} E_{2^{j}}\left(f^{\prime \prime}\right)_{p},
$$

with $E_{2^{j}}\left(f^{\prime \prime}\right)_{p}$ being the best approximation to $f^{\prime \prime}$ from $T_{2^{j}}$ in $L_{2 \pi}^{p}$. The constant $C_{p, j}$ depends only on $p$ for $1<p<\infty$, while an additional logarithmic factor $j$ appears for $p=1$ and $p=\infty$.

The proof of Theorem 2.3 is based on approximation-theoretical results not immediately connected to the wavelet context of this paper. Therefore, the relevant material is not presented here, but in the appendix. 
Some further remarks are appropriate at this point.

Remarks. 1. The first paper that describes nested spaces of trigonometric polynomials from a wavelet point of view is by C. K. Chui and H. N. Mhaskar [4]. The spaces $V_{j}$ in Definition 2.2 are in fact the very same ones (up to a shift of the indices by one) as those investigated in the Chui and Mhaskar paper, where only one scaling function is used to span $V_{j}$ as well as only one wavelet (instead of two, as shall be seen in the next section) to span the orthogonal complements. Furthermore, the scaling function in [4] gives rise to only a quasi-interpolation operator, and the corresponding wavelet also has no obvious interpolatory properties. This makes the construction of the relevant decomposition and reconstruction sequences more complicated. The Hermite interpolation operator in Definition 2.3 allows a simpler approach to the construction of the reconstruction and decomposition matrices (see $\S \S 4$ and 6$)$.

2. A Lagrange interpolation approach to trigonometric wavelets based on results by A. A. Privalov [14] was investigated by J. Prestin and E. Quak in [11, 12, 13]. The nested spaces generated by this method are different from the sample spaces $V_{j}$ of Definition 2.2. For the so-called de la Vallée Poussin interpolants, the trigonometric polynomials contained in the corresponding nested spaces satisfy a more intricate relation. For Fourier-type interpolants, however, the highest-degree polynomial is in fact a cosine term. For more details, see Theorem 7.1 in [11].

\section{INTERPOLATORY HERMITE-TYPE WAVELETS}

As the next step, the orthogonal complement $W_{j}$ of $V_{j}$ relative to $V_{j+1}$, i.e., the so-called wavelet space, needs to be described in more detail.

Definition 3.1 (wavelet functions). For $j \in \mathbb{N}_{0}$, define

$$
\psi_{j, 0}^{0}(x)=\frac{1}{2^{j+1}} \cos 2^{j+1} x+\frac{1}{3 \cdot 2^{2 j+1}} \sum_{\ell=2^{j+1}+1}^{2^{j+2}-1}\left(3 \cdot 2^{j+1}-\ell\right) \cos \ell x
$$

and

$$
\psi_{j, 0}^{1}(x)=\frac{1}{3 \cdot 2^{2 j+1}} \sum_{\ell=2^{j+1}+1}^{2^{j+2}-1} \sin \ell x+\frac{1}{2^{2 j+3}} \sin 2^{j+2} x .
$$

As for the scaling functions, for any $j \in \mathbb{N}_{0}$ and $n=0, \ldots, 2^{j+1}-1$, set $\psi_{j, n}^{0}(x)=$ $\psi_{j, 0}^{0}\left(x-x_{j, n}\right)$ and $\psi_{j, n}^{1}(x)=\psi_{j, 0}^{1}\left(x-x_{j, n}\right)$ with the same use of indices modulo $2^{j+1}$ as in Definition 2.1.

Definition 3.2. For $j \in \mathbb{N}_{0}$, the spaces $W_{j}$ are defined by

$$
W_{j}:=\operatorname{span}\left\{\psi_{j, n}^{0}, \psi_{j, n}^{1}: n=0, \ldots, 2^{j+1}-1\right\}
$$

Using Theorem 2.2, a careful inspection shows that $\psi_{j, 0}^{0}, \psi_{j, 0}^{1} \in V_{j+1}$, and a translation by $\frac{n \pi}{2^{j}}$ does not influence the terms $\cos 2^{j+1} x$ and $\sin 2^{j+2} x$, so that also for any $n=0, \ldots, 2^{j+1}-1$, it follows that

$$
\psi_{j, n}^{0}, \psi_{j, n}^{1} \in V_{j+1} .
$$


From the usual orthogonality properties of trigonometric polynomials, it is clear that

$$
\psi_{j, n}^{0}, \psi_{j, n}^{1} \perp V_{j},
$$

where orthogonality (denoted by $\perp$ ) is considered with respect to the usual inner product as introduced in $\S 2$. Since the cardinality of the spanning set for $W_{j}$ (i.e., $2^{j+2}$ ) is the same as the dimension of the relative orthogonal complement

$$
\operatorname{span}\left\{\cos 2^{j+1} x, \ldots, \cos \left(2^{j+2}-1\right) x, \sin \left(2^{j+1}+1\right) x, \ldots, \sin 2^{j+2} x\right\}
$$

of the space $V_{j}$ in $V_{j+1}$, it remains to verify the linear independence of the functions that span $W_{j}$. This will again be accomplished by using the interpolation properties.

In [11], following Privalov [14], we used a simple construction formula for the wavelet functions in terms of just one scaling function of the level $j+1$ and one of level $j$. In this Hermite case, the formulae are much more complicated.

Lemma 3.1. For $j \in \mathbb{N}_{0}$, the wavelets $\psi_{j, 0}^{0}$ and $\psi_{j, 0}^{1}$ have the following representation in terms of the scaling functions of level $j+1$ and $j$ :

$$
\begin{aligned}
\psi_{j, 0}^{0}= & \frac{4}{3} \phi_{j+1,0}^{0}-\frac{1}{3} \phi_{j, 0}^{0} \\
& +\frac{4}{3} \sum_{n=0}^{2^{j+1}-1} \cot \left(x_{j+2,2 n+1}\right) \phi_{j+1,2 n+1}^{1}-\frac{1}{3 \cdot 2^{j}} \sum_{n=0}^{2^{j+1}-1} \phi_{j+1,2 n+1}^{0}
\end{aligned}
$$

and

$$
\psi_{j, 0}^{1}=\frac{4}{3} \phi_{j+1,0}^{1}-\frac{1}{3} \phi_{j, 0}^{1}+\frac{1}{3 \cdot 2^{j}} \sum_{n=0}^{2^{j+1}-1} \phi_{j+1,2 n+1}^{1}
$$

Proof. Let us start with the expression in (3.4). Using the definitions (2.6) and (3.2), one finds directly

$$
\psi_{j, 0}^{1}(x)=\frac{4}{3} \phi_{j+1,0}^{1}(x)-\frac{1}{3} \phi_{j, 0}^{1}(x)+\frac{1}{3 \cdot 2^{2 j+3}}\left(\sin 2^{j+2} x-2 \sin 2^{j+1} x\right) .
$$

As the Hermite operator $L_{j+1}$ defined in Definition 2.3 is a projection on $V_{j+1}$, one obtains by direct computation

$$
\begin{aligned}
\frac{1}{3 \cdot 2^{2 j+3}}\left(\sin 2^{j+2} x-2 \sin 2^{j+1} x\right) & =\frac{1}{3 \cdot 2^{2 j+3}} L_{j+1}\left(\left(\sin 2^{j+2} \cdot-2 \sin 2^{j+1} \cdot\right)\right)(x) \\
& =\frac{1}{3 \cdot 2^{j}} \sum_{n=0}^{2^{j+1}-1} \phi_{j+1,2 n+1}^{1}(x)
\end{aligned}
$$

For (3.3), definitions (2.5) and (3.1) yield

$$
\begin{aligned}
\psi_{j, 0}^{0}(x)= & \frac{4}{3} \phi_{j+1,0}^{0}(x)-\frac{1}{3} \phi_{j, 0}^{0}(x) \\
& +\frac{1}{3 \cdot 2^{j}}\left(\sum_{\ell=2^{j+1}+1}^{2^{j+2}-1} \cos \ell x-\sum_{\ell=1}^{2^{j+1}-1} \cos \ell x\right)+\frac{1}{3 \cdot 2^{j+1}}\left(\cos 2^{j+1} x-1\right)
\end{aligned}
$$


As above, an application of the operator $L_{j+1}$ and some straightforward computation yields

$$
\begin{aligned}
\frac{1}{3 \cdot 2^{j+1}}\left(\cos 2^{j+1} x-1\right) & =\frac{1}{3 \cdot 2^{j+1}} L_{j+1}\left(\cos 2^{j+1} \cdot-1\right)(x) \\
& =-\frac{1}{3 \cdot 2^{j}} \sum_{n=0}^{2^{j+1}-1} \phi_{j+1,2 n+1}^{0}(x) .
\end{aligned}
$$

The remaining term can be dealt with in a similar way, but it is necessary to compute the values of the sums $\sum_{\ell=2^{j+1}+1}^{2^{j+2}} \cos \ell x$ and $\sum_{\ell=1}^{2^{j+1}-1} \cos \ell x$ and of their derivatives $\sum_{\ell=2^{j+1}+1}^{2^{j+2}-1}-\ell \sin \ell x$ and $\sum_{\ell=1}^{2^{j+1}-1}-\ell \sin \ell x$ at the knots $x_{j, n}$ and $x_{j+1,2 n+1}$, respectively. This can be performed by taking into account the explicit formula (2.1) for the Dirichlet kernel and its derivative, and yields, after some computations for $n=1, \ldots, 2^{j+1}-1$, the following results, which are recorded for later use:

$$
\begin{aligned}
\sum_{\ell=1}^{2^{j+1}-1} \cos \left(\ell x_{j, n}\right) & =\sum_{\ell=2^{j+1}+1}^{2^{j+2}-1} \cos \left(\ell x_{j, n}\right)=-1, \\
\sum_{\ell=1}^{2^{j+1}-1} \cos \left(\ell x_{j+1,2 n+1}\right) & =\sum_{\ell=2^{j+1}+1}^{2^{j+2}-1} \cos \left(\ell x_{j+1,2 n+1}\right)=0, \\
\sum_{\ell=1}^{2^{j+1}-1}-\ell \sin \left(\ell x_{j, n}\right) & =\sum_{\ell=2^{j+1}+1}^{2^{j+2}-1}-\ell \sin \left(\ell x_{j, n}\right)=2^{j} \cot \left(x_{j+1, n}\right),
\end{aligned}
$$

while

$$
\sum_{\ell=1}^{2^{j+1}-1}-\ell \sin \left(\ell x_{j+1,2 n+1}\right)=-2^{j} \cot \left(x_{j+2,2 n+1}\right)
$$

and

$$
\sum_{\ell=2^{j+1}+1}^{2^{j+2}-1}-\ell \sin \left(\ell x_{j+1,2 n+1}\right)=3 \cdot 2^{j} \cot \left(x_{j+2,2 n+1}\right) .
$$

These results imply the final equality

$$
\frac{1}{3 \cdot 2^{j}}\left(\sum_{\ell=2^{j+1}+1}^{2^{j+2}-1} \cos \ell x-\sum_{\ell=1}^{2^{j+1}-1} \cos \ell x\right)=\frac{4}{3} \sum_{n=0}^{2^{j+1}-1} \cot \left(x_{j+2,2 n+1}\right) \phi_{j+1,2 n+1}^{1}(x),
$$

which concludes the proof of this lemma.

Lemma 3.1 now enables us to state the interpolatory properties of the wavelets. 
Theorem 3.1 (Interpolatory properties of the wavelets). The following interpolatory properties hold for $k, n=0, \ldots, 2^{j+1}-1$, namely

$$
\psi_{j, n}^{0}\left(x_{j, k}\right)=\delta_{k, n} \quad \text { and } \quad \psi_{j, n}^{0}{ }^{\prime}\left(x_{j, k}\right)=0
$$

as well as

$$
\psi_{j, n}^{1}\left(x_{j, k}\right)=0 \quad \text { and } \quad \psi_{j, n}^{1}{ }^{\prime}\left(x_{j, k}\right)=\delta_{k, n} .
$$

The values at the interlacing points $x_{j+1,2 k+1}$ for $k=0, \ldots, 2^{j+1}-1$ are given by

$$
\begin{aligned}
& \psi_{j, n}^{0}\left(x_{j+1,2 k+1}\right)=-\frac{1}{3 \cdot 2^{j}}-\frac{1}{3 \cdot 2^{2 j+2}} \sin ^{-2}\left(x_{j+2,2 k+1-2 n}\right), \\
& \psi_{j, n}^{0}{ }^{\prime}\left(x_{j+1,2 k+1}\right)=\frac{4}{3} \cot \left(x_{j+2,2 k+1-2 n}\right)+\frac{1}{3 \cdot 2^{2 j+2}} \frac{\cot \left(x_{j+2,2 k+1-2 n}\right)}{\sin ^{2}\left(x_{j+2,2 k+1-2 n}\right)},
\end{aligned}
$$

while

$$
\begin{aligned}
\psi_{j, n}^{1}\left(x_{j+1,2 k+1}\right) & =-\frac{1}{3 \cdot 2^{2 j+1}} \cot \left(x_{j+2,2 k+1-2 n}\right), \\
\psi_{j, n}^{1}{ }^{\prime}\left(x_{j+1,2 k+1}\right) & =\frac{1}{3 \cdot 2^{j}}+\frac{1}{3 \cdot 2^{2 j+2}} \sin ^{-2}\left(x_{j+2,2 k+1-2 n}\right) .
\end{aligned}
$$

Proof. All these properties can be established by using the representations for the wavelets given in Lemma 3.1 and the interpolatory properties of the scaling functions of Theorem 2.1 .

As a first application, (3.10) and (3.11) imply the linear independence of all wavelet functions $\psi_{j, n}^{0}$ and $\psi_{j, n}^{1}$, and thus we have

Corollary 3.1. The space $W_{j}$ is the orthogonal complement of $V_{j}$ in $V_{j+1}$, i.e.,

$$
V_{j+1}=V_{j} \oplus W_{j}
$$

where $\oplus$ denotes orthogonal summation.

Remark. In [11], trigonometric Lagrange interpolants were investigated, where the corresponding wavelet functions interpolated the fundamental data set $\left\{\delta_{k, n}\right\}$ at the midpoints of the underlying partition. In fact, it can be shown that it is impossible to construct a wavelet function that is also a fundamental Lagrange interpolant on the original partition. The situation here, for Hermite interpolants, is strikingly different. Theorem 3.1 shows that the pair of functions in Definition 3.1 indeed interpolate the fundamental data of function and first derivative values at the points of the given original partition.

Thus, the scaling function $\phi_{j, 0}^{0}$ is a low-frequency fundamental interpolant of function data, while $\psi_{j, 0}^{0}$ is a high-frequency fundamental interpolant at the same points (and analogously for the first derivative for $\phi_{j, 0}^{1}$ and $\psi_{j, 0}^{1}$ ). Figures 1 and 2 show the functions $\phi_{4,16}^{0}, \phi_{4,16}^{1}$ and $\psi_{4,16}^{0}$ and $\psi_{4,16}^{1}$, respectively.

Note also that while the scaling function $\phi_{j, 0}^{0}$ is even and the scaling function $\phi_{j, 0}^{1}$ is odd, this is also true for the corresponding wavelets, namely $\psi_{j, 0}^{0}$ is even and $\psi_{j, 0}^{1}$ is odd. 
TRIGONOMETRIC HERMITE WAVELETS
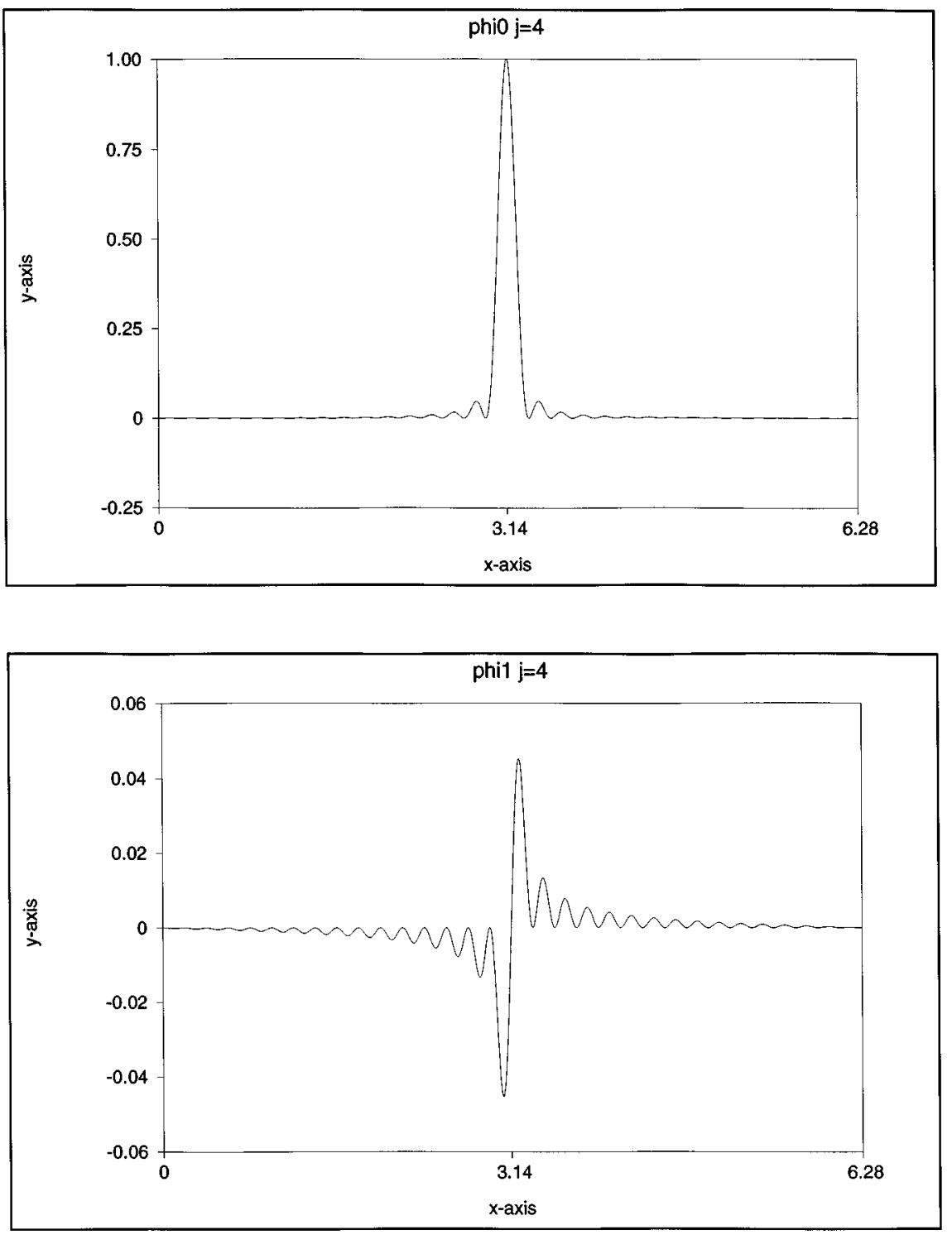

Figure 1 

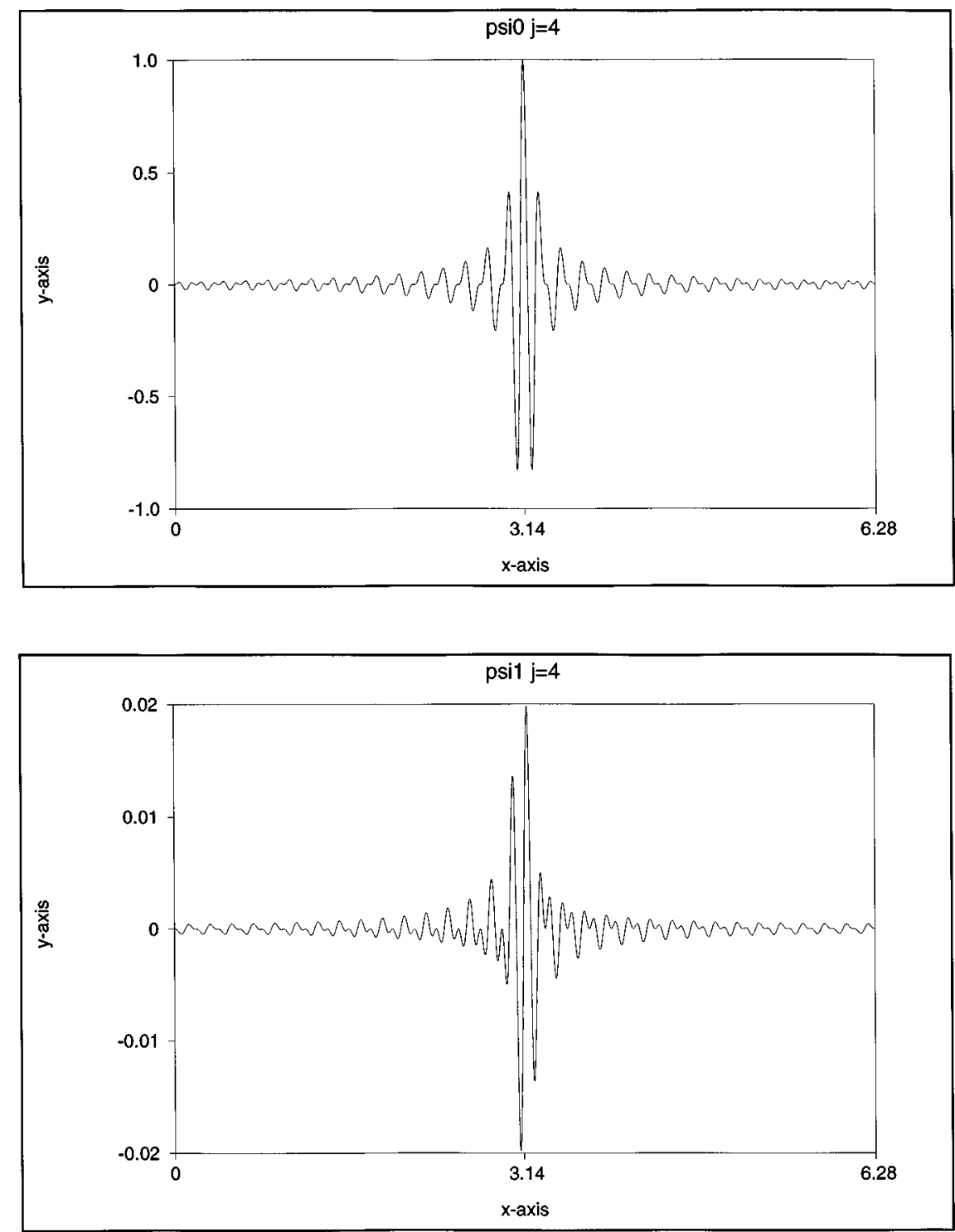

FIGURE 2

\section{TWO-SCALE RELATIONS}

The availability of the Hermite interpolation operator facilitates the computation of the finite two-scale sequences considerably as compared to the corresponding treatment of the two-scale sequences of the spaces $V_{j}$ in [4]. In the Lagrange case (see [11]), a reconstruction matrix consisting of four circulant blocks was computed. Here, since we have two scaling functions and two wavelets, there will be sixteen such circulant blocks. Its particular entries are already known by applying Theorems 2.1 and 3.1 . 
As $V_{j} \subset V_{j+1}$, there have to exist specific coefficients $p_{j, n, s}^{0,0}$ and $p_{j, n, s}^{0,1}$ such that $\phi_{j, n}^{0}=\sum_{s}\left(p_{j, n, s}^{0,0} \phi_{j+1, s}^{0}+p_{j, n, s}^{0,1} \phi_{j+1, s}^{1}\right)$, as well as coefficients $p_{j, n, s}^{1,0}$ and $p_{j, n, s}^{1,1}$ such that $\phi_{j, n}^{1}=\sum_{s}\left(p_{j, n, s}^{1,0} \phi_{j+1, s}^{0}+p_{j, n, s}^{1,1} \phi_{j+1, s}^{1}\right)$. The following result establishes their precise values.

Theorem 4.1. For $j \in \mathbb{N}_{0}$ and $n=0,1 \ldots, 2^{j+1}-1$, we have

$$
\begin{aligned}
\phi_{j, n}^{0}= & \phi_{j+1,2 n}^{0}+\sum_{s=0}^{2^{j+1}-1} \phi_{j, n}^{0}\left(x_{j+1,2 s+1}\right) \phi_{j+1,2 s+1}^{0}+\sum_{s=0}^{2^{j+1}-1} \phi_{j, n}^{0}{ }^{\prime}\left(x_{j+1,2 s+1}\right) \phi_{j+1,2 s+1}^{1} \\
= & \phi_{j+1,2 n}^{0}+\sum_{s=0}^{2^{j+1}-1} \frac{1}{2^{2 j+2}} \sin ^{-2}\left(x_{j+2,2 s+1-2 n}\right) \phi_{j+1,2 s+1}^{0} \\
& +\sum_{s=0}^{2^{j+1}-1}-\frac{1}{2^{2 j+2}} \frac{\cot \left(x_{j+2,2 s+1-2 n}\right)}{\sin ^{2}\left(x_{j+2,2 s+1-2 n}\right)} \phi_{j+1,2 s+1}^{1}
\end{aligned}
$$

and

$$
\begin{aligned}
\phi_{j, n}^{1}= & \phi_{j+1,2 n}^{1}+\sum_{s=0}^{2^{j+1}-1} \phi_{j, n}^{1}\left(x_{j+1,2 s+1}\right) \phi_{j+1,2 s+1}^{0}+\sum_{s=0}^{2^{j+1}-1} \phi_{j, n}^{1}{ }^{\prime}\left(x_{j+1,2 s+1}\right) \phi_{j+1,2 s+1}^{1} \\
= & \phi_{j+1,2 n}^{0}+\sum_{s=0}^{2^{j+1}-1} \frac{1}{2^{2 j+1}} \cot \left(x_{j+2,2 s+1-2 n}\right) \phi_{j+1,2 s+1}^{0} \\
& +\sum_{s=0}^{2^{j+1}-1}-\frac{1}{2^{2 j+2}} \sin ^{-2}\left(x_{j+2,2 s+1-2 n}\right) \phi_{j+1,2 s+1}^{1} .
\end{aligned}
$$

Proof. Using once again the fact that the interpolatory operator $L_{j+1}$ in Definition 2.3 is a projection for $V_{j+1}$, we find that

$$
\phi_{j, n}^{0}(x)=L_{j+1}\left(\phi_{j, n}^{0}\right)(x)=\sum_{k=0}^{2^{j+2}-1} \phi_{j, n}^{0}\left(x_{j+1, k}\right) \phi_{j+1, k}^{0}(x)+\phi_{j, n}^{0}{ }^{\prime}\left(x_{j+1, k}\right) \phi_{j+1, k}^{1}(x)
$$

and

$$
\phi_{j, n}^{1}(x)=\sum_{k=0}^{2^{j+2}-1} \phi_{j, n}^{1}\left(x_{j+1, k}\right) \phi_{j+1, k}^{0}(x)+\phi_{j, n}^{1}{ }^{\prime}\left(x_{j+1, k}\right) \phi_{j+1, k}^{1}(x) .
$$

The interpolatory properties of Theorem 2.1 now imply the desired results.

Analogously, from $W_{j} \subset V_{j+1}$, it is clear that there have to be coefficients $q_{j, n, s}^{0,0}$ and $q_{j, n, s}^{0,1}$ such that $\psi_{j, n}^{0}=\sum_{s}\left(q_{j, n, s}^{0,0} \phi_{j+1, s}^{0}+q_{j, n, s}^{0,1} \phi_{j+1, s}^{1}\right)$, as well as coefficients $q_{j, n, s}^{1,0}$ and $q_{j, n, s}^{1,1}$ such that $\psi_{j, n}^{1}=\sum_{s}\left(q_{j, n, s}^{1,0} \phi_{j+1, s}^{0}+q_{j, n, s}^{1,1} \phi_{j+1, s}^{1}\right)$. These coefficients are determined in the following theorem. 
Theorem 4.2. For $j \in \mathbb{N}_{0}$ and $n=0,1 \ldots, 2^{j+1}-1$, we have

$$
\begin{aligned}
\psi_{j, n}^{0}= & \phi_{j+1,2 n}^{0}+\sum_{s=0}^{2^{j+1}-1} \psi_{j, n}^{0}\left(x_{j+1,2 s+1}\right) \phi_{j+1,2 s+1}^{0}+\sum_{s=0}^{2^{j+1}-1} \psi_{j, n}^{0}{ }^{\prime}\left(x_{j+1,2 s+1}\right) \phi_{j+1,2 s+1}^{1} \\
= & \phi_{j+1,2 n}^{0}+\sum_{s=0}^{2^{j+1}-1}\left(-\frac{1}{3 \cdot 2^{j}}-\frac{1}{3 \cdot 2^{2 j+2}} \sin ^{-2}\left(x_{j+2,2 s+1-2 n}\right)\right) \phi_{j+1,2 s+1}^{0} \\
& +\sum_{s=0}^{2^{j+1}-1}\left(\frac{4}{3} \cot \left(x_{j+2,2 s+1-2 n}\right)+\frac{1}{3 \cdot 2^{2 j+2}} \frac{\cot \left(x_{j+2,2 s+1-2 n}\right)}{\sin ^{2}\left(x_{j+2,2 s+1-2 n}\right)}\right) \phi_{j+1,2 s+1}^{1}
\end{aligned}
$$

and

$$
\begin{aligned}
\psi_{j, n}^{1}= & \phi_{j+1,2 n}^{1}+\sum_{s=0}^{2^{j+1}-1} \psi_{j, n}^{1}\left(x_{j+1,2 s+1}\right) \phi_{j+1,2 s+1}^{0}+\sum_{s=0}^{2^{j+1}-1} \psi_{j, n}^{1}{ }^{\prime}\left(x_{j+1,2 s+1}\right) \phi_{j+1,2 s+1}^{1} \\
= & \phi_{j+1,2 n}^{0}-\sum_{s=0}^{2^{j+1}-1} \frac{1}{3 \cdot 2^{2 j+1}} \cot \left(x_{j+2,2 s+1-2 n}\right) \phi_{j+1,2 s+1}^{0} \\
& +\sum_{s=0}^{2^{j+1}-1}\left(\frac{1}{3 \cdot 2^{j}}+\frac{1}{3 \cdot 2^{2 j+2}} \sin ^{-2}\left(x_{j+2,2 s+1-2 n}\right)\right) \phi_{j+1,2 s+1}^{1} .
\end{aligned}
$$

Proof. As in Theorem 4.1 above, the projection property of $L_{j+1}$ and the interpolatory properties of the functions $\psi_{j, n}^{0}$ and $\psi_{j, n}^{1}$ from Theorem 3.1 are the necessary ingredients for the proof.

Let $\Phi_{j}^{0}$ denote the vector $\left(\phi_{j, 0}^{0}, \phi_{j, 1}^{0}, \ldots, \phi_{j, 2^{j+1}-1}^{0}\right)^{T}$, and let us also introduce $\Phi_{j}^{1}=\left(\phi_{j, 0}^{1}, \phi_{j, 1}^{1}, \ldots, \phi_{j, 2^{j+1}-1}^{1}\right)^{T}, \Psi_{j}^{0}=\left(\psi_{j, 0}^{0}, \psi_{j, 1}^{0}, \ldots, \psi_{j, 2^{j+1}-1}^{0}\right)^{T}$, and finally $\Psi_{j}^{1}=$ $\left(\psi_{j, 0}^{1}, \psi_{j, 1}^{1}, \ldots, \psi_{j, 2^{j+1}-1}^{1}\right)^{T}$.

Furthermore, we define a reordering for the vector of scaling functions (either for 0 or 1) by

$$
\begin{aligned}
P_{j} \Phi_{j+1}= & \left(\phi_{j+1,0}, \phi_{j+1,2}, \ldots, \phi_{j+1,2 m}, \ldots, \phi_{j+1,2^{j+2}-2},\right. \\
& \left.\phi_{j+1,1}, \phi_{j+1,3}, \ldots, \phi_{j+1,2 m+1}, \ldots, \phi_{j+1,2^{j+2}-1}\right)^{T}
\end{aligned}
$$

i.e., $P_{j}$ is chosen to be the suitable permutation matrix for this ordering. Then Theorems 4.1 and 4.2 can be expressed as

$$
\left(\begin{array}{c}
\Phi_{j}^{0} \\
\Phi_{j}^{1} \\
\Psi_{j}^{0} \\
\Psi_{j}^{1}
\end{array}\right)=C_{j}\left(\begin{array}{c}
P_{j} \Phi_{j+1}^{0} \\
P_{j} \Phi_{j+1}^{1}
\end{array}\right)
$$

Here, the two-scale relation or reconstruction matrix $C_{j}$ has the following form:

$$
C_{j}=\left(\begin{array}{cccc}
I_{j} & 2^{-2 j-2} S_{j} & 0 & -2^{-2 j-2} U_{j} \\
0 & 2^{-2 j-1} T_{j} & I_{j} & -2^{-2 j-2} S_{j} \\
I_{j} & -\frac{1}{3}\left(2^{-j} E_{j}+2^{-2 j-2} S_{j}\right) & 0 & \frac{1}{3}\left(4 T_{j}+2^{-2 j-2} U_{j}\right) \\
0 & -\frac{1}{3} 2^{-2 j-1} T_{j} & I_{j} & \frac{1}{3}\left(2^{-j} E_{j}+2^{-2 j-2} S_{j}\right)
\end{array}\right)
$$


where for each block the subscript $j$ indicates a submatrix of dimension $2^{j+1}$. In fact, all these submatrices are circulant (see the monograph of Davis [6] for a comprehensive treatment of circulant matrices) and, consequently, in implementations it is possible to use efficient techniques such as Fast Fourier Transforms. According to Theorems 4.1 and $4.2, I_{j}$ is the identity matrix, $S_{j}=\left(\sin ^{-2}\left(x_{j+2,2 s+1-2 n}\right)\right)_{n, s}$, $T_{j}=\left(\cot \left(x_{j+2,2 s+1-2 n}\right)\right)_{n, s}, U_{j}=\left(\frac{\cot \left(x_{j+2,2 s+1-2 n}\right)}{\sin ^{2}\left(x_{j+2,2 s+1-2 n}\right)}\right)_{n, s}$ and $E_{j}=(1)_{n, s}$ the matrix with constant entries one. Thus, $C_{j}$ is a square matrix of dimension $2^{j+3}$ with 16 circulant blocks.

As a consequence of Corollary 3.1, both the sets $\left\{\phi_{j, r}^{0}, \phi_{j, r}^{1}, \psi_{j, r}^{0}, \psi_{j, r}^{1}\right\}_{r=0}^{2^{j+1}-1}$ and $\left\{\phi_{j+1, r}^{0}, \phi_{j+1, r}^{1}\right\}_{r=0}^{2^{j+2}-1}$ are bases of $V_{j+1}$. Therefore, as it represents a change of basis, the reconstruction matrix $C_{j}$ is nonsingular and its inverse $D_{j}$ is the decomposition matrix such that

$$
\left(\begin{array}{c}
P_{j} \Phi_{j+1}^{0} \\
P_{j} \Phi_{j+1}^{1}
\end{array}\right)=D_{j}\left(\begin{array}{c}
\Phi_{j}^{0} \\
\Phi_{j}^{1} \\
\Psi_{j}^{0} \\
\Psi_{j}^{1}
\end{array}\right) .
$$

In order to obtain a detailed description of the entries of $D_{j}$, it becomes necessary to investigate inner products of scaling functions and to introduce dual scaling functions.

\section{INNER PRODUCTS OF SCALING FUNCTIONS AND DUAL FUNCTIONS}

In this section, dual scaling functions, and thus the inner products of the functions $\phi_{j, k}^{0}$ and $\phi_{j, k}^{1}$, are studied in more detail to eventually facilitate the computation of the decomposition matrix $D_{j}$ in $\S 6$.

Definition 5.1. For any $j \in \mathbb{N}_{0}$, the functions $\tilde{\phi}_{j, r}^{0}$ and $\tilde{\phi}_{j, r}^{1} \in V_{j}$, for $r=$ $0, \ldots, 2^{j+1}-1$, uniquely determined by the conditions

$$
\left\langle\tilde{\phi}_{j, r}^{0}, \phi_{j, k}^{0}\right\rangle=\delta_{r, k}, \quad\left\langle\tilde{\phi}_{j, r}^{0}, \phi_{j, k}^{1}\right\rangle=0
$$

and

$$
\left\langle\tilde{\phi}_{j, r}^{1}, \phi_{j, k}^{0}\right\rangle=0, \quad\left\langle\tilde{\phi}_{j, r}^{1}, \phi_{j, k}^{1}\right\rangle=\delta_{r, k},
$$

respectively, for all $r, k=0, \ldots, 2^{j+1}-1$, are called dual scaling functions (or duals of the functions $\left.\phi_{j, r}^{0}, \phi_{j, r}^{1}\right)$.

Note that the dual scaling functions lie in the same space $V_{j}$ as the original scaling functions. Consequently, the dual functions can be written as linear combinations of these scaling functions. It is a well-known fact that the coefficients in these linear combinations are nothing but the entries of the inverse matrix of the inner product matrix of the scaling functions. Specifically, for any $j \in \mathbb{N}_{0}$, we have

$$
\tilde{\phi}_{j, r}^{0}=\sum_{s=0}^{2^{j+1}-1}\left(\alpha_{j, r, s}^{0,0} \phi_{j, s}^{0}+\alpha_{j, r, s}^{0,1} \phi_{j, s}^{1}\right)
$$

and

$$
\tilde{\phi}_{j, r}^{1}=\sum_{s=0}^{2^{j+1}-1}\left(\alpha_{j, r, s}^{1,0} \phi_{j, s}^{0}+\alpha_{j, r, s}^{1,1} \phi_{j, s}^{1}\right),
$$


where the duality conditions lead to a linear system of equations for each dual function $\tilde{\phi}_{j, r}^{i}, r=0, \ldots, 2^{j+1}-1, i=0,1$, namely,

$$
\sum_{s=0}^{2^{j+1}-1} \alpha_{j, r, s}^{i, 0}\left\langle\phi_{j, k}^{\tilde{i}}, \phi_{j, s}^{0}\right\rangle+\alpha_{j, r, s}^{i, 1}\left\langle\phi_{j, k}^{\tilde{i}}, \phi_{j, s}^{1}\right\rangle=\delta_{r, k} \cdot \delta_{i, \tilde{i}}, \quad k=0, \ldots, 2^{j+1}-1, \tilde{i}=0,1 .
$$

Its coefficient matrix is always the inner product matrix

$$
G_{j}=\left(\begin{array}{cc}
G_{j}^{0,0} & G_{j}^{0,1} \\
G_{j}^{1,0} & G_{j}^{1,1}
\end{array}\right)
$$

where

$$
\begin{aligned}
& G_{j}^{0,0}=\left(\left\langle\phi_{j, k}^{0}, \phi_{j, s}^{0}\right\rangle\right)_{k, s=0}^{2^{j+1}-1}, \quad G_{j}^{0,1}=\left(\left\langle\phi_{j, k}^{0}, \phi_{j, s}^{1}\right\rangle\right)_{k, s=0}^{2^{j+1}-1}, \\
& G_{j}^{1,0}=\left(\left\langle\phi_{j, k}^{1}, \phi_{j, s}^{0}\right\rangle\right)_{k, s=0}^{2^{j+1}-1}, \quad G_{j}^{1,1}=\left(\left\langle\phi_{j, k}^{1}, \phi_{j, s}^{1}\right\rangle\right)_{k, s=0}^{2^{j+1}-1},
\end{aligned}
$$

and different right-hand sides correspond to different dual functions. Note that, of course, $G_{j}^{1,0}=G_{j}^{0,1^{T}}$.

Therefore, the next step must be a closer investigation of the inner products of the scaling functions. This is done in the following lemma, which also implies that the scaling functions are not mutually orthogonal.

Lemma 5.1. The inner products of the scaling functions on level $j \in \mathbb{N}_{0}$ are

$$
\begin{aligned}
& \left\langle\phi_{j, k}^{0}, \phi_{j, s}^{0}\right\rangle= \begin{cases}\frac{1}{3}\left(\frac{1}{2^{j}}+\frac{1}{2^{3 j+3}}\right), & k=s, \\
\frac{1}{2^{3 j+3}} \sin ^{-2}\left(x_{j+1, k-s}\right), & k \neq s,\end{cases} \\
& \left\langle\phi_{j, k}^{0}, \phi_{j, s}^{1}\right\rangle= \begin{cases}0, & k=s, \\
\frac{1}{2^{3 j+3}} \cot \left(x_{j+1, k-s}\right), & k \neq s,\end{cases} \\
& \left\langle\phi_{j, k}^{1}, \phi_{j, s}^{1}\right\rangle= \begin{cases}\frac{1}{2^{3 j+2}}-\frac{3}{2^{4 j+5}}, & k=s, \\
-\frac{3}{2^{4 j+5}}, & k \neq s .\end{cases}
\end{aligned}
$$

Proof. First, observe that after an appropriate substitution, it suffices to consider inner products of the form $\left\langle\phi_{j, 0}, \phi_{j, s}\right\rangle$. Secondly, it is possible to use the representation formulae in (2.3) for the Dirichlet kernel and (2.4) for the conjugate Dirichlet kernel to facilitate the evaluation of the inner products.

The simplest case is (5.3), where (2.4) can be used to establish

$$
\begin{aligned}
& \left\langle\phi_{j, 0}^{1}, \phi_{j, s}^{1}\right\rangle=\frac{1}{2^{4 j+2}}\left\langle\tilde{D}_{2^{j+1}-1}(\cdot)+\frac{1}{2} \sin 2^{j+1}(\cdot), \tilde{D}_{2^{j+1}-1}\left(\cdot-x_{j, s}\right)+\frac{1}{2} \sin 2^{j+1}(\cdot)\right\rangle \\
& =\frac{1}{2^{4 j+2}}\left(\left\langle\tilde{D}_{2^{j+1}-1}(\cdot), \tilde{D}_{2^{j+1}-1}\left(\cdot-x_{j, s}\right)\right\rangle+\frac{1}{4}\left\langle\frac{1}{2} \sin 2^{j+1}(\cdot), \frac{1}{2} \sin 2^{j+1}(\cdot)\right\rangle\right) \\
& \quad=\frac{1}{2^{4 j+2}}\left(-\frac{1}{2} \tilde{S}_{2^{j+1}-1}\left(\tilde{D}_{2^{j+1}-1}\right)\left(x_{j, s}\right)+\frac{1}{8}\right) \\
& \quad=\frac{1}{2^{4 j+2}}\left(\frac{1}{2} \sum_{\ell=1}^{2^{j+1}-1} \cos \left(\ell x_{j, s}\right)+\frac{1}{8}\right) .
\end{aligned}
$$


Here, the summation formula (3.5) for $s \neq 0$ and a direct computation for $s=0$ are used.

In order to prove (5.2), we consider

$$
\begin{aligned}
& \left\langle\phi_{j, 0}^{0}, \phi_{j, s}^{1}\right\rangle=\frac{1}{2^{4 j+2}}\left\langle\sum_{\ell=0}^{2^{j+1}-1} D_{\ell}(\cdot), \tilde{D}_{2^{j+1}-1}\left(\cdot-x_{j, s}\right)+\frac{1}{2} \sin 2^{j+1}(\cdot)\right\rangle \\
& =\frac{1}{2^{4 j+2}} \sum_{\ell=0}^{2^{j+1}-1}\left\langle D_{\ell}(\cdot), \tilde{D}_{2^{j+1}-1}\left(\cdot-x_{j, s}\right)\right\rangle=\frac{1}{2^{4 j+2}} \sum_{\ell=0}^{2^{j+1}-1}-\frac{1}{2} \tilde{S}_{2^{j+1}-1}\left(\tilde{D}_{\ell}\right)\left(x_{j, s}\right) \\
& =-\frac{1}{2^{4 j+3}} \sum_{\ell=1}^{2^{j+1}-1} \sum_{r=1}^{\ell} \sin \left(r x_{j, s}\right)=-\frac{1}{2^{4 j+3}} \sum_{\ell=1}^{2^{j+1}-1}\left(2^{j+1}-\ell\right) \sin \left(\ell x_{j, s}\right) \\
& =\frac{1}{2^{4 j+3}} \sum_{\ell=1}^{2^{j+1}-1} \ell \sin \left(\ell x_{j, s}\right)
\end{aligned}
$$

by using (2.12), so that for $s \neq 0,(3.7)$ yields (5.2).

Finally, by using (2.1), the Fejér kernel (2.5) can be rewritten as

$$
\frac{1}{2^{2 j+1}} \sum_{\ell=0}^{2^{j+1}-1} D_{\ell}(x)=\frac{1}{2^{j+1}}+\frac{1}{2^{2 j+1}} \sum_{\ell=1}^{2^{j+1}-1}\left(2^{j+1}-\ell\right) \cos \ell x .
$$

Thus, we have

$$
\begin{aligned}
& \left\langle\phi_{j, 0}^{0}, \phi_{j, s}^{0}\right\rangle=\left\langle\frac{1}{2^{j+1}}+\frac{1}{2^{2 j+1}} \sum_{\ell=1}^{2^{j+1}-1}\left(2^{j+1}-\ell\right) \cos \ell \cdot \frac{1}{2^{2 j+1}} \sum_{r=0}^{2^{j+1}-1} D_{r}\left(\cdot-x_{j, s}\right)\right\rangle \\
& =\frac{1}{2^{3 j+2}} \sum_{r=0}^{2^{j+1}-1}\left\langle 1, D_{r}\left(\cdot-x_{j, s}\right)\right\rangle \\
& \quad+\frac{1}{2^{4 j+2}} \sum_{\ell=1}^{2^{j+1}-1}\left(2^{j+1}-\ell\right) \sum_{r=0}^{2^{j+1}-1}\left\langle\cos \ell \cdot, D_{r}\left(\cdot-x_{j, s}\right)\right\rangle \\
& =\frac{1}{2^{2 j+2}}+\frac{1}{2^{4 j+3}} \sum_{\ell=1}^{2^{j+1}-1}\left(2^{j+1}-\ell\right) \sum_{r=0}^{2^{j+1}-1} S_{r}(\cos \ell \cdot)\left(x_{j, s}\right) \\
& =\frac{1}{2^{2 j+2}}+\frac{1}{2^{4 j+3}} \sum_{\ell=1}^{2^{j+1}-1}\left(2^{j+1}-\ell\right)^{2} \cos \left(\ell x_{j, s}\right) \\
& =\frac{1}{2^{2 j+2}}+\frac{1}{2^{4 j+3}} \sum_{\ell=1}^{2^{j+1}-1} \ell^{2} \cos \left(\ell x_{j, s}\right) .
\end{aligned}
$$

For $s=0$, a direct computation gives (5.1), so that it remains to establish that, for $s \neq 0$,

$$
\sum_{\ell=1}^{2^{j+1}-1} \ell^{2} \cos \left(\ell x_{j, s}\right)=2^{j} \sin ^{-2}\left(x_{j+1, s}\right)-2^{2 j+1} .
$$


This is accomplished by starting from the formula (2.1), namely

$$
2 \sin \frac{x}{2} D_{2^{j+1}-1}(x)=\sin \left(2^{j+1}-\frac{1}{2}\right) x .
$$

Taking the second derivative yields

$$
\begin{aligned}
2 & \sin \frac{x}{2} D^{\prime \prime}{ }_{2^{j+1}-1}(x)+2 \cos \frac{x}{2} D_{2^{j+1}-1}^{\prime}(x)-\frac{1}{2} \sin \frac{x}{2} D_{2^{j+1}-1}(x) \\
& =-\left(2^{j+1}-\frac{1}{2}\right)^{2} \sin \left(2^{j+1}-\frac{1}{2}\right) x .
\end{aligned}
$$

The formulae (3.5) and (3.7) give the values of $D_{2^{j+1}-1}\left(x_{j, s}\right)$ and $D^{\prime}{ }_{2 j+1}-1\left(x_{j, s}\right)$, so that the result of an evaluation of (5.5) at the knots $x_{j, s}$ can be rewritten to produce

$$
D^{\prime \prime}{ }_{2+1}^{j+1}\left(x_{j, s}\right)=-2^{j} \cot ^{2}\left(x_{j+1, s}\right)-2^{j}+2^{2 j+1},
$$

which implies (5.4).

The interpolatory properties of the functions $\phi_{j, k}^{0}$ and $\phi_{j, k}^{1}$ allow us to establish some results on the matrices $G_{j}^{0,0}, G_{j}^{0,1}, G_{j}^{1,0}$ and $G_{j}^{1,1}$ which turn out to be useful when it comes to the computation of the inverse of $G_{j}$.

Lemma 5.2. For $j \in \mathbb{N}_{0}$, the row sums of the matrices $G_{j}^{0,0}, G_{j}^{0,1}=G_{j}^{1,0^{T}}$ and $G_{j}^{1,1}$ are

$$
\begin{aligned}
\sum_{\ell=0}^{2^{j+1}-1}\left(G_{j}^{0,0}\right)_{k, \ell} & =\frac{1}{2^{j+1}}, \\
\sum_{\ell=0}^{2^{j+1}-1}\left(G_{j}^{0,1}\right)_{k, \ell} & =0, \\
\sum_{\ell=0}^{2^{j+1}-1}\left(G_{j}^{1,1}\right)_{k, \ell} & =\frac{1}{2^{3 j+4}},
\end{aligned}
$$

for arbitrary $k=0, \ldots, 2^{j+1}-1$. By using the matrix $E_{j}=(1)_{k, s=0}^{2^{j+1}-1}$ with constant entries 1, we can express this as

$$
G_{j}^{0,0} E_{j}=\frac{1}{2^{j+1}} E_{j}, \quad G_{j}^{0,1} E_{j}=0 \quad \text { and } \quad G_{j}^{1,1} E_{j}=\frac{1}{2^{3 j+4}} E_{j} .
$$

Proof. As all matrices are circulant, only the first rows have to be investigated. Using the fact that the constant function 1 is interpolated exactly at each level $j$, we obtain

$$
\sum_{\ell=0}^{2^{j+1}-1} \phi_{j, \ell}^{0}=1
$$

and thus

$$
\sum_{\ell=0}^{2^{j+1}-1}\left(G_{j}^{0,0}\right)_{0, \ell}=\left\langle\phi_{j, 0}^{0}, \sum_{\ell=0}^{2^{j+1}-1} \phi_{j, \ell}^{0}\right\rangle=\frac{1}{2^{2 j+1}} \frac{1}{2 \pi} \int_{0}^{2 \pi} \sum_{\ell=0}^{2^{j+1}-1} D_{\ell}(t) d t=\frac{1}{2^{j+1}} .
$$


To prove (5.7), we make use of the following fact:

$$
\frac{1}{2^{j+1}} \sin 2^{j+1} x=\frac{1}{2^{j+1}} L_{j}\left(\sin 2^{j+1} \cdot\right)(x)=\sum_{\ell=0}^{2^{j+1}-1} \phi_{j, \ell}^{1} .
$$

Therefore, we have

$$
\sum_{\ell=0}^{2^{j+1}-1}\left(G_{j}^{0,1}\right)_{0, \ell}=\left\langle\phi_{j, 0}^{0}, \sum_{\ell=0}^{2^{j+1}-1} \phi_{j, \ell}^{1}\right\rangle=\frac{1}{2^{3 j+2}}\left\langle\sum_{\ell=0}^{2^{j+1}-1} D_{\ell}(\cdot), \sin 2^{j+1}(\cdot)\right\rangle=0 .
$$

Finally, (5.8) is merely a consequence of some direct computation.

By using the values of the matrix entries in (5.1) and (5.2), we have the following

Corollary 5.1. For $j \in \mathbb{N}_{0}$, the formulae (5.6) and (5.7) can be written as

$$
\sum_{\ell=1}^{2^{j+1}-1} \sin ^{-2}\left(x_{j+1, \ell}\right)=\frac{1}{3}\left(2^{2 j+2}-1\right)
$$

and

$$
\sum_{\ell=1}^{2^{j+1}-1} \cot \left(x_{j+1, \ell}\right)=0
$$

Consequently, it follows that

$$
\sum_{\ell=1}^{2^{j+1}-1} \sin ^{-2}\left(x_{j+2,2 \ell+1}\right)=2^{2 j+2}
$$

and

$$
\sum_{\ell=1}^{2^{j+1}-1} \cot \left(x_{j+2,2 \ell+1}\right)=0
$$

The final step before the computation of $G_{j}^{-1}$ is to find a way to rewrite $G_{j}^{0,1} \cdot G_{j}^{0,1}$.

Lemma 5.3. For any $j \in \mathbb{N}_{0}$, we have

$$
G_{j}^{0,1} \cdot G_{j}^{0,1}=\frac{1}{2^{4 j+4}} I_{j}+\frac{1}{2^{5 j+5}} E_{j}-\frac{1}{2^{3 j+2}} G_{j}^{0,0} .
$$

Proof. First, the diagonal elements $\left(G_{j}^{0,1} \cdot G_{j}^{0,1}\right)_{k, k}$ for any $k=0, \ldots, 2^{j+1}-1$ according to the representation (5.2) of the elements of $G_{j}^{0,1}$ are

$$
\begin{aligned}
& \frac{1}{2^{6 j+6}} \sum_{\ell=0, \ell \neq k}^{2^{j+1}-1} \cot \left(x_{j+1, k-\ell}\right) \cot \left(x_{j+1, \ell-k}\right) \\
& =-\frac{1}{2^{6 j+6}} \sum_{\ell=0, \ell \neq k}^{2^{j+1}-1}\left(\sin ^{-2}\left(x_{j+1, k-\ell}\right)-1\right) \\
& =\frac{1}{2^{5 j+5}}-\frac{1}{2^{6 j+6}}-\frac{1}{2^{6 j+6}} \sum_{\ell=1}^{2^{j+1}-1} \sin ^{-2}\left(x_{j+1, \ell}\right) \\
& =\frac{1}{2^{4 j+4}}+\frac{1}{2^{5 j+5}}-\frac{1}{2^{3 j+2}}\left(\frac{1}{3 \cdot 2^{j}}+\frac{1}{3 \cdot 2^{3 j+3}}\right) \quad(\text { by }(5.9)) .
\end{aligned}
$$


For the off-diagonal elements, i.e., $k \neq s$, it follows that

$$
\left(G_{j}^{0,1} \cdot G_{j}^{0,1}\right)_{k, s}=\frac{1}{2^{6 j+6}} \sum_{\ell=0, \ell \neq k, \ell \neq s}^{2^{j+1}-1} \cot \left(x_{j+1, k-\ell}\right) \cot \left(x_{j+1, \ell-s}\right) .
$$

The formula

$$
\cot \alpha \cdot \cot \beta=\cot (\alpha+\beta)[\cot \alpha+\cot \beta]+1
$$

(for appropriate values of $\alpha$ and $\beta$ ) yields

$$
\begin{aligned}
& \left(G_{j}^{0,1} \cdot G_{j}^{0,1}\right)_{k, s} \\
& =\frac{1}{2^{6 j+6}} \sum_{\ell=0, \ell \neq k, \ell \neq s}^{2^{j+1}-1}\left\{\cot \left(x_{j+1, k-s}\right)\left[\cot \left(x_{j+1, k-\ell}\right)+\cot \left(x_{j+1, \ell-s}\right)\right]+1\right\} \\
& =\frac{1}{2^{6 j+6}} \cot \left(x_{j+1, k-s}\right)\left[-2 \cot \left(x_{j+1, k-s}\right)\right]+\frac{1}{2^{5 j+5}}-\frac{1}{2^{6 j+5}},
\end{aligned}
$$

where (5.10) has been used twice for the sums of cotangents.

Now, the inverse of the inner product matrix $G_{j}$ can be described in some detail.

Theorem 5.1. The inverse of the inner product matrix $G_{j}$ of the scaling functions, and therefore the coefficient matrix of the dual scaling functions, is given by

$$
G_{j}^{-1}=\left(\begin{array}{ll}
\alpha_{j}^{0,0} & \alpha_{j}^{0,1} \\
\alpha_{j}^{1,0} & \alpha_{j}^{1,1}
\end{array}\right)
$$

where

$$
\begin{array}{rlrl}
\alpha_{j}^{0,0} & =2^{j+2} I_{j}-E_{j}, & & \alpha_{j}^{0,1}=-2^{4 j+4} G_{j}^{0,1}, \\
\alpha_{j}^{1,0}=-2^{4 j+4} G_{j}^{1,0}=2^{4 j+4} G_{j}^{0,1} & \text { and } & \alpha_{j}^{1,1}=2^{4 j+4} G_{j}^{0,0}+2^{2 j+2} E_{j} .
\end{array}
$$

Here, as above, $I_{j}$ is an identity matrix, $E_{j}$ a matrix with constant entries 1, and the matrices $G_{j}^{0,0}, G_{j}^{0,1}$ and $G_{j}^{1,0}$ are the inner product matrices introduced in Lemma 5.1. All of these matrices are circulant square matrices of dimension $2^{j+1}$.

Proof. The proof is obtained by a direct computation and an investigation of the four matrix equations stemming from

$$
\left(\begin{array}{ll}
G_{j}^{0,0} & G_{j}^{0,1} \\
G_{j}^{1,0} & G_{j}^{1,1}
\end{array}\right)\left(\begin{array}{ll}
\alpha_{j}^{0,0} & \alpha_{j}^{0,1} \\
\alpha_{j}^{1,0} & \alpha_{j}^{1,1}
\end{array}\right)=\left(\begin{array}{cc}
I_{j} & 0 \\
0 & I_{j}
\end{array}\right)
$$

First, by using the definitions for the $\alpha$-terms, the matrix version of (5.6), and Lemma 5.3, we have

$$
G_{j}^{0,0} \alpha_{j}^{0,0}+G_{j}^{0,1} \alpha_{j}^{1,0}=2^{j+2} G_{j}^{0,0}-G_{j}^{0,0} E_{j}+2^{4 j+4} G_{j}^{0,1} \cdot G_{j}^{0,1}=I_{j} .
$$


Secondly, by applying the matrix version of (5.7) and taking into account that circulant matrices commute $[6, \mathrm{p} .68]$, we have

$$
G_{j}^{0,0} \alpha_{j}^{0,1}+G_{j}^{0,1} \alpha_{j}^{1,1}=-2^{4 j+4} G_{j}^{0,0} \cdot G_{j}^{0,1}+2^{4 j+4} G_{j}^{0,1} \cdot G_{j}^{0,0}+2^{2 j+2} G_{j}^{0,1} E_{j}=0 .
$$

For the other two steps, the simple structure of $G_{j}^{1,1}=\frac{1}{2^{3 j+2}} I_{j}-\frac{3}{2^{4 j+5}} E_{j}$ is used. In the third equation, this along with the definitions of the $\alpha$-terms, yields

$$
G_{j}^{1,0} \alpha_{j}^{0,0}+G_{j}^{1,1} \alpha_{j}^{1,0}=2^{j+2} G_{j}^{1,0}-G_{j}^{1,0} E_{j}-2^{j+2} G_{j}^{1,0}+\frac{3}{2} E_{j} G_{j}^{1,0}=0,
$$

where (5.7) and the fact that the column sums of circulant matrices are identical to the row sums, i.e., $E_{j} G_{j}^{1,0}=0$, are also used.

Finally, using that $G_{j}^{1,0}=-G_{j}^{0,1}$, Lemma 5.3, (5.6) for column sums and $E_{j}^{2}=$ $2^{j+1} E_{j}$, we have

$$
\begin{aligned}
G_{j}^{1,0} & \alpha_{j}^{0,1}+G_{j}^{1,1} \alpha_{j}^{1,1} \\
& =-2^{4 j+4} G_{j}^{1,0} \cdot G_{j}^{0,1}+2^{j+2} G_{j}^{0,0}+\frac{1}{2^{j}} E_{j}-\frac{3}{2} E_{j} G_{j}^{0,0}-\frac{3}{2^{2 j+3}} E_{j}^{2}=I_{j} .
\end{aligned}
$$

Again, some remarks seem to be in order.

Remarks. 1. The circulant structure of the $\alpha$-matrices in Theorem 5.1 can be used to show (much like the Lagrange case in [12]) that the dual functions are also translates by multiples of $\frac{\pi}{2^{j}}$ of the functions $\tilde{\phi}_{j, 0}^{0}$ and $\tilde{\phi}_{j, 0}^{1}$, just as is the case for the original scaling functions.

2. The wavelets in the Hermite multiresolution analysis are not mutually orthogonal on a given level $j$, but of course there is orthogonality between scales, i.e., by Chui $[3$, p.15] the wavelets are semiorthogonal.

Along the same lines as presented in $\$ 5$ for scaling functions, it is also possible to define dual wavelet functions as an alternative basis of the wavelet spaces $W_{j}$. In order to do so, the inner products of the wavelet functions need to be computed, again leading to a matrix $H_{j}$ with four circulant blocks. Analogously, the coefficients of the dual wavelets would then be the entries of the inverse matrix of $H_{j}$.

As the dual wavelets are not necessary for the purpose of finding the decomposition matrices (see the following section), the relevant computations are not described in this paper.

At the end of this section, we will consider inner products of scaling functions on consecutive levels, which form another building block for the subsequent detailed computation of the entries of the decomposition matrix.

Lemma 5.4. The inner products of scaling functions on levels $j \in \mathbb{N}_{0}$ and $j+1$ for $k=0, \ldots, 2^{j+1}-1$ and $s=0, \ldots, 2^{j+2}-1$ are given by

$$
\left\langle\phi_{j, k}^{0}, \phi_{j+1, s}^{0}\right\rangle= \begin{cases}\frac{1}{3}\left(\frac{1}{2^{3 j+5}}+\frac{5}{2^{j+3}}\right), & 2 k=s \\ \frac{1}{2^{3 j+5}} \sin ^{-2}\left(x_{j+2, s-2 k}\right), & 2 k \neq s, \text { s even } \\ \frac{1}{2^{3 j+4}} \sin ^{-2}\left(x_{j+2, s-2 k}\right), & s \text { odd }\end{cases}
$$




$$
\begin{aligned}
& \left\langle\phi_{j, k}^{0}, \phi_{j+1, s}^{1}\right\rangle= \begin{cases}0, & 2 k=s, \\
-\frac{1}{2^{3 j+5}} \cot \left(x_{j+2, s-2 k}\right), & 2 k \neq s,\end{cases} \\
& \left\langle\phi_{j, k}^{1}, \phi_{j+1, s}^{0}\right\rangle= \begin{cases}0, & 2 k=s, \\
\frac{1}{2^{3 j+5}} \cot \left(x_{j+2, s-2 k}\right), & 2 k \neq s, s \text { even }, \\
\frac{3}{2^{3 j+5}} \cot \left(x_{j+2, s-2 k}\right), & s \text { odd },\end{cases} \\
& \left\langle\phi_{j, k}^{1}, \phi_{j+1, s}^{1}\right\rangle= \begin{cases}\frac{1}{2^{3 j+4}}-\frac{1}{2^{4 j+6}}, & 2 k=s, \\
-\frac{1}{2^{4 j+6}}, & 2 k \neq s .\end{cases}
\end{aligned}
$$

Proof. Similar to Lemma 5.1, and because of $\left\langle\phi_{j, k}, \phi_{j+1, s}\right\rangle=\left\langle\phi_{j, 0}, \phi_{j+1, s-2 k}\right\rangle$, all statements will be proved for the case $k=0$. Also, as in Lemma 5.1, the computations will rely on the representations (2.3) and (2.4).

First, we have

$$
\begin{aligned}
& \left\langle\phi_{j, 0}^{0}, \phi_{j+1, s}^{0}\right\rangle=\frac{1}{2^{4 j+4}}\left\langle 2^{j}+\sum_{\ell=1}^{2^{j+1}-1}\left(2^{j+1}-\ell\right) \cos \ell \cdot \sum_{r=0}^{2^{j+2}-1} D_{r}\left(\cdot-x_{j+1, s}\right)\right\rangle \\
& =\frac{1}{2^{2 j+3}}+\frac{1}{2^{4 j+4}} \sum_{\ell=1}^{2^{j+1}-1}\left(2^{j+1}-\ell\right) \sum_{r=0}^{2^{j+2}-1} \frac{1}{2} S_{r}(\cos \ell \cdot)\left(x_{j+1, s}\right) \\
& =\frac{1}{2^{2 j+3}}+\frac{1}{2^{4 j+5}} \sum_{\ell=1}^{2^{j+1}-1}\left(2^{j+1}-\ell\right)\left(2^{j+2}-\ell\right) \cos \left(\ell x_{j+1, s}\right) \\
& =\frac{1}{2^{2 j+3}}+\frac{1}{2^{3 j+4}} \sum_{\ell=1}^{2^{j+1}-1}\left(2^{j+1}-\ell\right) \cos \left(\ell x_{j+1, s}\right)+\frac{1}{2^{4 j+5}} \sum_{\ell=1}^{2^{j+1}-1}\left(2^{j+1}-\ell\right)^{2} \cos \left(\ell x_{j+1, s}\right) \\
& =\frac{1}{2^{2 j+3}}+\frac{1}{2^{3 j+4}}(-1)^{s} \sum_{\ell=1}^{2^{j+1}-1} \ell \cos \left(\ell x_{j+1, s}\right)+\frac{1}{2^{4 j+5}}(-1)^{s} \sum_{\ell=1}^{2^{j+1}-1} \ell^{2} \cos \left(\ell x_{j+1, s}\right) .
\end{aligned}
$$

The case $s=0$ follows by a straightforward computation. To evaluate the cosine sums, note that

$$
\begin{aligned}
\sum_{\ell=1}^{2^{j+1}-1} \ell \cos \left(\ell x_{j+1, s}\right) & =2^{2 j+1} \phi_{j, 0}^{1}{ }^{\prime}\left(x_{j+1, s}\right)-2^{j}(-1)^{s} \\
& = \begin{cases}-\frac{1}{2} \sin ^{-2}\left(x_{j+1, s}\right)+2^{j}, & s \text { odd, } \\
-2^{j}, & s \text { even, } s \neq 0 .\end{cases}
\end{aligned}
$$

Furthermore, we have

$$
\sum_{\ell=1}^{2^{j+1}-1} \ell^{2} \cos \left(\ell x_{j+1, s}\right)=-D_{2^{j+1}-1}^{\prime \prime}\left(x_{j+1, s}\right)
$$


and evaluation of formula (5.5) at the knots $x_{j+1, s}$ gives

$$
D_{2^{j+1}-1}^{\prime \prime}\left(x_{j+1, s}\right)=(-1)^{s}\left(2^{2 j+1}-2^{j} \sin ^{-2}\left(x_{j+2, s}\right)\right) .
$$

Combining these results yields (5.14).

Secondly, we have

$$
\begin{aligned}
& \left\langle\phi_{j, 0}^{0}, \phi_{j+1, s}^{1}\right\rangle=\frac{1}{2^{4 j+4}}\left\langle\sum_{\ell=0}^{2^{j+1}-1} D_{\ell}(\cdot), \tilde{D}_{2^{j+1}-1}\left(\cdot-x_{j+1, s}\right)+\frac{1}{2} \sin 2^{j+2}(\cdot)\right\rangle \\
& =-\frac{1}{2^{4 j+5}} \sum_{\ell=0}^{2^{j+1}-1} \tilde{S}_{2^{j+2}-1}\left(D_{\ell}\right)\left(x_{j+1, s}\right)=-\frac{1}{2^{4 j+5}} \sum_{\ell=0}^{2^{j+1}-1}\left(2^{j+1}-\ell\right) \sin \left(\ell x_{j+1, s}\right) \\
& =-\frac{1}{2^{4 j+5}}(-1)^{s} D_{2^{j+1}-1}^{\prime}\left(x_{j+1, s}\right) .
\end{aligned}
$$

From here, the case $s=0$ is immediate, while taking the first derivative of the closed formula for the Dirichlet kernel and evaluating it at $x_{j+1, s}$ takes care of the rest of $(5.15)$.

Thirdly, we have

$$
\begin{aligned}
\left\langle\phi_{j, 0}^{1}, \phi_{j+1, s}^{0}\right\rangle & =\frac{1}{2^{4 j+4}}\left\langle\tilde{D}_{2^{j+1}-1}(\cdot)+\frac{1}{2} \sin 2^{j+1}(\cdot), \sum_{\ell=0}^{2^{j+2}-1} D_{\ell}\left(\cdot-x_{j+1, s}\right)\right\rangle \\
& =\frac{1}{2^{4 j+5}}\left(\sum_{\ell=1}^{2^{j+1}-1}+\sum_{\ell=2^{j+1}}^{2^{j+2}-1}\right) S_{\ell}\left(\tilde{D}_{2^{j+1}-1}(\cdot)+\frac{1}{2} \sin 2^{j+1}(\cdot)\right)\left(x_{j+1, s}\right) \\
& =\frac{1}{2^{4 j+5}}\left(\sum_{\ell=1}^{2^{j+1}-1}\left(2^{j+1}-\ell\right) \sin \left(\ell x_{j+1, s}\right)+2^{j+1} \tilde{D}_{2^{j+1}-1}\left(x_{j+1, s}\right)\right) \\
& =\frac{1}{2^{4 j+5}}\left((-1)^{s} \sum_{\ell=1}^{2^{j+1}-1}-\ell \sin \left(\ell x_{j+1, s}\right)+2^{3 j+2} \phi_{j, 0}^{1}\left(x_{j+1, s}\right)\right)
\end{aligned}
$$

Thus, from (3.8) and the interpolatory properties (2.12) and (2.15), one obtains (5.16).

Finally, we have

$$
\begin{aligned}
& \left\langle\phi_{j, 0}^{1}, \phi_{j+1, s}^{1}\right\rangle \\
& =\frac{1}{2^{4 j+4}}\left\langle\tilde{D}_{2^{j+1}-1}(\cdot)+\frac{1}{2} \sin 2^{j+1}(\cdot), \tilde{D}_{2^{j+2}-1}\left(\cdot-x_{j+1, s}\right)+\frac{1}{2} \sin 2^{j+2}(\cdot)\right\rangle \\
& =-\frac{1}{2^{4 j+5}} \tilde{S}_{2^{j+2}-1}\left(\tilde{D}_{2^{j+1}-1}(\cdot)+\frac{1}{2} \sin 2^{j+1}(\cdot)\right)\left(x_{j+1, s}\right) \\
& =\frac{1}{2^{4 j+5}}\left(D_{2^{j+1}-1}\left(x_{j+1, s}\right)-\frac{1}{2}+\frac{1}{2}(-1)^{s}\right) .
\end{aligned}
$$

Evaluation of formula (2.1) now settles (5.17). 


\section{Decomposition SEQuences}

Since $V_{j+1}=V_{j} \oplus W_{j}$ for $j \in \mathbb{N}_{0}$, any $\phi_{j+1, n}^{0}$ and $\phi_{j+1, n}^{1} \in V_{j+1}$ can be written as a linear combination of the basis functions of $V_{j}$ and $W_{j}$, i.e., $\phi_{j, k}^{0}, \phi_{j, k}^{1}, \psi_{j, k}^{0}$ and $\psi_{j, k}^{1}$. The computation of the relevant coefficients will not be performed by inverting the reconstruction matrix, but instead, by using the dual functions and the interpolatory properties of the scaling functions and the wavelets.

Theorem 6.1. For any $j \in \mathbb{N}_{0}$ and $m=0, \ldots, 2^{j+1}-1$, we have

$$
\phi_{j+1,2 m}^{0}=\sum_{s=0}^{2^{j+1}-1}\left(a_{j, m, s}^{0,0} \phi_{j, s}^{0}+a_{j, m, s}^{0,1} \phi_{j, s}^{1}+b_{j, m, s}^{0,0} \psi_{j, s}^{0}+b_{j, m, s}^{0,1} \psi_{j, s}^{1}\right),
$$

where for $s=0, \ldots, 2^{j+1}-1$ the decomposition coefficients are given by

$$
\begin{aligned}
a_{j, m, s}^{0,0} & =\frac{3}{4} \delta_{m, s}-\frac{1}{2^{j+3}}, \\
a_{j, m, s}^{0,1} & = \begin{cases}0, & m=s, \\
-\frac{1}{4} \cot \left(x_{j+1, m-s}\right), & m \neq s,\end{cases} \\
b_{j, m, s}^{0,0} & =\frac{1}{4} \delta_{m, s}+\frac{1}{2^{j+3}}, \\
b_{j, m, s}^{0,1} & = \begin{cases}0, & m=s, \\
\frac{1}{4} \cot \left(x_{j+1, m-s}\right), & m \neq s .\end{cases}
\end{aligned}
$$

Proof. The general approach for the proof of this theorem and the ones that follow will always be the same. The coefficients for the scaling functions are determined by taking inner products with the corresponding dual functions, then by using the formulae for the dual coefficients and the inner products of Lemma 5.4. The wavelet coefficients can then be derived simply by using the interpolatory properties of scaling functions and wavelets from Theorems 2.1 and 3.1.

Starting from the representation

$$
\phi_{j+1,2 m}^{0}=\sum_{k=0}^{2^{j+1}-1}\left(a_{j, m, k}^{0,0} \phi_{j, k}^{0}+a_{j, m, k}^{0,1} \phi_{j, k}^{1}+b_{j, m, k}^{0,0} \psi_{j, k}^{0}+b_{j, m, k}^{0,1} \psi_{j, k}^{1}\right),
$$

we take inner products with the dual function $\tilde{\phi}_{j, s}^{0} \in V_{j} \perp W_{j}$, which results according to Definition 5.1 - in

$$
\begin{aligned}
& a_{j, m, s}^{0,0}=\left\langle\tilde{\phi}_{j, s}^{0}, \phi_{j+1,2 m}^{0}\right\rangle=\sum_{\ell=0}^{2^{j+1}-1}\left(\alpha_{j, s, \ell}^{0,0}\left\langle\phi_{j, \ell}^{0}, \phi_{j+1,2 m}^{0}\right\rangle+\alpha_{j, s, \ell}^{0,1}\left\langle\phi_{j, \ell}^{1}, \phi_{j+1,2 m}^{0}\right\rangle\right) \\
& =\sum_{\ell=0}^{2^{j+1}-1}\left(2^{j+2} \delta_{s, \ell}-1\right)\left\langle\phi_{j, \ell}^{0}, \phi_{j+1,2 m}^{0}\right\rangle-2^{j+1} \sum_{\ell=0, \ell \neq s}^{2^{j+1}-1} \cot \left(x_{j+1, s-\ell}\right)\left\langle\phi_{j, \ell}^{1}, \phi_{j+1,2 m}^{0}\right\rangle \\
& =2^{j+2}\left\langle\phi_{j, 0}^{0}, \phi_{j+1,2 m-2 s}^{0}\right\rangle-\left\langle 1, \phi_{j+1,2 m}^{0}\right\rangle \\
& \quad-2^{j+1} \sum_{\ell=0, \ell \neq s}^{2^{j+1}-1} \cot \left(x_{j+1, s-\ell}\right)\left\langle\phi_{j, 0}^{1}, \phi_{j+1,2 m-2 \ell}^{0}\right\rangle \\
& =2^{j+2}\left\langle\phi_{j, 0}^{0}, \phi_{j+1,2 m-2 s}^{0}\right\rangle-\frac{1}{2^{j+2}}-\frac{1}{2^{2 j+4}} \sum_{\ell=0, \ell \neq s, \ell \neq m}^{2^{j+1}-1} \cot \left(x_{j+1, s-\ell}\right) \cot \left(x_{j+1, m-\ell}\right),
\end{aligned}
$$


by using the results of Theorem 5.1 and Lemma 5.4 in the process. For the case $m=s$, this yields

$$
\frac{5}{6}+\frac{1}{3 \cdot 2^{2 j+3}}-\frac{1}{2^{j+2}}-\frac{1}{2^{2 j+4}} \sum_{\ell=1}^{2^{j+1}-1}\left(\sin ^{-2}\left(x_{j+1, \ell}\right)-1\right)
$$

and thus by (5.9) the desired term. For $m \neq s$, one obtains

$$
\frac{1}{2^{2 j+3}} \sin ^{-2}\left(x_{j+1, m-s}\right)-\frac{1}{2^{j+2}}+\frac{1}{2^{2 j+4}} \sum_{\ell=0, \ell \neq s, \ell \neq m}^{2^{j+1}-1} \cot \left(x_{j+1, \ell-s}\right) \cot \left(x_{j+1, m-\ell}\right),
$$

and then one handles the cotangent sum as in the proof of Lemma 5.3.

To compute $a_{j, m, s}^{0,1}$, the inner product is taken with $\tilde{\phi}_{j, s}^{1}$, producing

$$
\begin{aligned}
a_{j, m, s}^{0,1}= & \left\langle\tilde{\phi}_{j, s}^{1}, \phi_{j+1,2 m}^{0}\right\rangle \\
= & 2^{j+1} \sum_{\ell=0, \ell \neq s}^{2^{j+1}-1} \cot \left(x_{j+1, s-\ell}\right)\left(\frac{1}{2^{3 j+5}} \sin ^{-2}\left(x_{j+1, m-\ell}\right)\left(1-\delta_{\ell, m}\right)\right. \\
& \left.+\delta_{\ell, m} \frac{1}{3}\left(\frac{1}{2^{3 j+5}}+\frac{5}{2^{j+3}}\right)\right) \\
& +\frac{1}{2^{2 j+4}} \sum_{\ell=0, \ell \neq s, \ell \neq m}^{2^{j+1}-1} \sin ^{-2}\left(x_{j+1, s-\ell}\right) \cot \left(x_{j+1, m-\ell}\right) \\
& +2^{j-1} \frac{1}{3}\left(\frac{1}{2^{j}}+\frac{1}{2^{3 j+3}}\right) \cot \left(x_{j+1, m-s}\right)\left(1-\delta_{s, m}\right)+2^{2 j+2}\left\langle\sum_{\ell=0}^{2^{j+1}-1} \phi_{j, \ell}^{1}, \phi_{j+1,2 m}^{0}\right\rangle \\
= & 2^{j+1} \cot \left(x_{j+1, s-m}\right) \frac{1}{3}\left(\frac{1}{2^{3 j+5}}+\frac{5}{2^{j+3}}\right)\left(1-\delta_{s, m}\right) \\
& +2^{j-1} \frac{1}{3}\left(\frac{1}{2^{j}}+\frac{1}{2^{3 j+3}}\right) \cot \left(x_{j+1, m-s}\right)\left(1-\delta_{s, m}\right)+2^{j+1}\left\langle\sin 2^{j+1} \cdot, \phi_{j+1,2 m}^{0}\right\rangle .
\end{aligned}
$$

For the wavelet coefficients, evaluation of the decomposition equation at the knots $x_{j, s}$ and the use of the interpolatory properties from Theorems 2.1 and 3.1 give the equation $\delta_{s, m}=a_{j, m, s}^{0,0}+b_{j, m, s}^{0,0}$, while taking the derivative followed by evaluation at the knots $x_{j, s}$ results in $0=a_{j, m, s}^{0,1}+b_{j, m, s}^{0,1}$.

Theorem 6.2. For any $j \in \mathbb{N}_{0}$ and $m=0, \ldots, 2^{j+1}-1$, we have

$$
\phi_{j+1,2 m+1}^{0}=\sum_{s=0}^{2^{j+1}-1}\left(\tilde{a}_{j, m, s}^{0,0} \phi_{j, s}^{0}+\tilde{a}_{j, m, s}^{0,1} \phi_{j, s}^{1}+\tilde{b}_{j, m, s}^{0,0} \psi_{j, s}^{0}+\tilde{b}_{j, m, s}^{0,1} \psi_{j, s}^{1}\right),
$$

where for $s=0, \ldots, 2^{j+1}-1$ the decomposition coefficients are given by

$$
\begin{aligned}
& \tilde{a}_{j, m, s}^{0,0}=\frac{1}{2^{j+3}}+\frac{1}{2^{2 j+4}} \sin ^{-2}\left(x_{j+2,2 m+1-2 s}\right), \\
& \tilde{a}_{j, m, s}^{0,1}=\frac{1}{4} \cot \left(x_{j+2,2 m+1-2 s}\right)+\frac{1}{2^{2 j+4}} \frac{\cot \left(x_{j+2,2 m+1-2 s}\right)}{\sin ^{2}\left(x_{j+2,2 m+1-2 s}\right)}, \\
& \tilde{b}_{j, m, s}^{0,0}=-\frac{1}{2^{j+3}}-\frac{1}{2^{2 j+4}} \sin ^{-2}\left(x_{j+2,2 m+1-2 s}\right), \\
& \tilde{b}_{j, m, s}^{0,1}=-\frac{1}{4} \cot \left(x_{j+2,2 m+1-2 s}\right)-\frac{1}{2^{2 j+4}} \frac{\cot \left(x_{j+2,2 m+1-2 s}\right)}{\sin ^{2}\left(x_{j+2,2 m+1-2 s}\right)} .
\end{aligned}
$$


Proof. As before, we start with taking inner products of the dual function $\tilde{\phi}_{j, s}^{0}$ in the equation

$$
\phi_{j+1,2 m+1}^{0}=\sum_{k=0}^{2^{j+1}-1}\left(\tilde{a}_{j, m, k}^{0,0} \phi_{j, k}^{0}+\tilde{a}_{j, m, k}^{0,1} \phi_{j, k}^{1}+\tilde{b}_{j, m, k}^{0,0} \psi_{j, k}^{0}+\tilde{b}_{j, m, k}^{0,1} \psi_{j, k}^{1}\right),
$$

to obtain, by using Theorem 5.1 and Lemma 5.4,

$$
\begin{aligned}
\tilde{a}_{j, m, s}^{0,0}= & \left\langle\tilde{\phi}_{j, s}^{0}, \phi_{j+1,2 m+1}^{0}\right\rangle=2^{j+2}\left\langle\phi_{j, s}^{0}, \phi_{j+1,2 m+1}^{0}\right\rangle \\
& -\left\langle\sum_{\ell=0}^{2^{j+1}-1} \phi_{j, \ell}^{0}, \phi_{j+1,2 m+1}^{0}\right\rangle-2^{j+1} \sum_{\ell=0, \ell \neq s}^{2^{j+1}-1} \cot \left(x_{j+1, s-\ell}\right)\left\langle\phi_{j, \ell}^{1}, \phi_{j+1,2 m+1}^{0}\right\rangle \\
= & \frac{1}{2^{2 j+2}} \sin ^{-2}\left(x_{j+2,2 m+1-2 s}\right)-\frac{1}{2^{j+2}} \\
& -\frac{3}{2^{2 j+4}} \sum_{\ell=0, \ell \neq s}^{2^{j+1}-1} \cot \left(x_{j+1, s-\ell}\right) \cot \left(x_{j+2,2 m+1-2 \ell}\right) .
\end{aligned}
$$

Appealing to the cotangent formula (5.13) leads to

$$
\begin{aligned}
\tilde{a}_{j, m, s}^{0,0}= & \frac{1}{2^{j+3}}-\frac{3}{2^{2 j+4}}+\frac{1}{2^{2 j+2}} \sin ^{-2}\left(x_{j+2,2 m+1-2 s}\right) \\
& +\frac{3}{2^{2 j+4}} \cot \left(x_{j+2,2 m+1-2 s}\right) \sum_{\ell=0, \ell \neq s}^{2^{j+1}-1}\left(\cot \left(x_{j+1, \ell-s}\right)+\cot \left(x_{j+2,2 m+1-2 \ell}\right)\right) .
\end{aligned}
$$

In view of (5.10) and (5.12), the cotangent sums collapse to a single term, namely $-\cot \left(x_{j+2,2 m+1-2 s}\right)$, and rewriting everything in sine terms yields the desired result.

Inner products with the dual function $\tilde{\phi}_{j, s}^{1}$ now give

$$
\begin{aligned}
\tilde{a}_{j, m, s}^{0,1}=\left\langle\tilde{\phi}_{j, s}^{1}, \phi_{j+1,2 m+1}^{0}\right\rangle= & \frac{1}{2^{2 j+3}} \sum_{\ell=0, \ell \neq s}^{2^{j+1}-1} \cot \left(x_{j+1, s-\ell}\right) \sin ^{-2}\left(x_{j+2,2 m+1-2 \ell}\right) \\
& +\frac{3}{2^{2 j+4}} \sum_{\ell=0, \ell \neq s}^{2^{j+1}-1} \cot \left(x_{j+2,2 m+1-2 \ell}\right) \sin ^{-2}\left(x_{j+1, s-\ell}\right) \\
& +\left(\frac{1}{2}+\frac{1}{2^{2 j+4}}\right) \cot \left(x_{j+2,2 m+1-2 s}\right) .
\end{aligned}
$$

Rewriting this expression in cotangent terms and applying (5.13) for both sums as well as (5.10) and (5.12) results in

$$
\begin{aligned}
\tilde{a}_{j, m, s}^{0,1}= & \frac{1}{2^{2 j+3}} \sum_{\ell=0, \ell \neq s}^{2^{j+1}-1} \cot \left(x_{j+1, s-\ell}\right) \cot ^{2}\left(x_{j+2,2 m+1-2 \ell}\right) \\
& +\frac{1}{2^{2 j+3}} \sum_{\ell=0, \ell \neq s}^{2^{j+1}-1} \cot \left(x_{j+1, s-\ell}\right)
\end{aligned}
$$




$$
\begin{aligned}
& +\frac{3}{2^{2 j+4}} \sum_{\ell=0, \ell \neq s}^{2^{j+1}-1} \cot \left(x_{j+2,2 m+1-2 \ell}\right) \cot ^{2}\left(x_{j+1, s-\ell}\right) \\
& +\frac{3}{2^{2 j+4}} \sum_{\ell=0, \ell \neq s}^{2^{j+1}-1} \cot \left(x_{j+2,2 m+1-2 \ell}\right)+\left(\frac{1}{2}+\frac{1}{2^{2 j+4}}\right) \cot \left(x_{j+2,2 m+1-2 s}\right) \\
& =-\frac{1}{2^{2 j+3}} \sum_{\ell=0, \ell \neq s}^{2^{j+1}-1} \cot \left(x_{j+2,2 m+1-2 \ell}\right)\left[\operatorname { c o t } ( x _ { j + 2 , 2 m + 1 - 2 s } ) \left(\cot \left(x_{j+1, \ell-s}\right)\right.\right. \\
& \left.\left.+\cot \left(x_{j+2,2 m+1-2 \ell}\right)\right)+1\right] \\
& +\frac{3}{2^{2 j+4}} \sum_{\ell=0, \ell \neq s}^{2^{j+1}-1} \cot \left(x_{j+1, \ell-s}\right)\left[\operatorname { c o t } ( x _ { j + 2 , 2 m + 1 - 2 s } ) \left(\cot \left(x_{j+1, \ell-s}\right)\right.\right. \\
& +\left(\frac{1}{2}-\frac{1}{2^{2 j+3}}\right) \cot \left(x_{j+2,2 m+1-2 s}\right) \\
& \left.\left.+\cot \left(x_{j+2,2 m+1-2 \ell}\right)\right)+1\right] \\
& =\frac{1}{2} \cot \left(x_{j+2,2 m+1-2 s}\right) \\
& +\frac{1}{2^{2 j+4}} \cot \left(x_{j+2,2 m+1-2 s}\right) \sum_{\ell=0, \ell \neq s}^{2^{j+1}-1} \cot \left(x_{j+1, \ell-s}\right) \cot \left(x_{j+2,2 m+1-2 \ell}\right) \\
& -\frac{1}{2^{2 j+3}} \cot \left(x_{j+2,2 m+1-2 s}\right) \sum_{\ell=0, \ell \neq s}^{2^{j+1}-1} \cot ^{2}\left(x_{j+2,2 m+1-2 \ell}\right) \\
& +\frac{3}{2^{2 j+4}} \cot \left(x_{j+2,2 m+1-2 s}\right) \sum_{\ell=0, \ell \neq s}^{2^{j+1}-1} \cot ^{2}\left(x_{j+1, \ell-s}\right) \\
& =\frac{1}{2} \cot \left(x_{j+2,2 m+1-2 s}\right)+\frac{1}{2^{2 j+4}}\left(2^{j+1}-1\right) \cot \left(x_{j+2,2 m+1-2 s}\right) \\
& +\frac{1}{2^{2 j+4}} \cot ^{2}\left(x_{j+2,2 m+1-2 s}\right) \sum_{\ell=0, \ell \neq s}^{2^{j+1}-1}\left(\cot \left(x_{j+1, \ell-s}\right)+\cot \left(x_{j+2,2 m+1-2 \ell}\right)\right) \\
& -\frac{1}{2^{2 j+3}} \cot \left(x_{j+2,2 m+1-2 s}\right) \sum_{\ell=0, \ell \neq s}^{2^{j+1}-1}\left(\sin ^{-2}\left(x_{j+2,2 m+1-2 \ell}\right)-1\right) \\
& +\frac{3}{2^{2 j+4}} \cot \left(x_{j+2,2 m+1-2 s}\right) \sum_{\ell=0, \ell \neq s}^{2^{j+1}-1}\left(\sin ^{-2}\left(x_{j+1, \ell-s}\right)-1\right) \\
& =\frac{1}{2} \cot \left(x_{j+2,2 m+1-2 s}\right)-\frac{1}{2^{2 j+4}} \cot ^{3}\left(x_{j+2,2 m+1-2 s}\right) \\
& -\frac{1}{2^{2 j+3}} \cot \left(x_{j+2,2 m+1-2 s}\right) \sum_{\ell=0, \ell \neq s}^{2^{j+1}-1} \sin ^{-2}\left(x_{j+2,2 m+1-2 \ell}\right) \\
& +\frac{3}{2^{2 j+4}} \cot \left(x_{j+2,2 m+1-2 s}\right) \sum_{\ell=0, \ell \neq s}^{2^{j+1}-1} \sin ^{-2}\left(x_{j+2, \ell-s}\right) .
\end{aligned}
$$


Formulae (5.9) and (5.11) from Corollary 5.1 complete this step.

The wavelet terms are determined just as for Theorem 6.1 by evaluating the decomposition formula and its derivative at the knots $x_{j, s}$.

Theorem 6.3. For any $j \in \mathbb{N}_{0}$ and $m=0, \ldots, 2^{j+1}-1$, we have

$$
\phi_{j+1,2 m}^{1}=\sum_{s=0}^{2^{j+1}-1}\left(a_{j, m, s}^{1,0} \phi_{j, s}^{0}+a_{j, m, s}^{1,1} \phi_{j, s}^{1}+b_{j, m, s}^{1,0} \psi_{j, s}^{0}+b_{j, m, s}^{1,1} \psi_{j, s}^{1}\right),
$$

where for $s=0, \ldots, 2^{j+1}-1$ the decomposition coefficients are given by

$$
\begin{array}{ll}
a_{j, m, s}^{1,0}=0, & a_{j, m, s}^{1,1}=\frac{1}{4} \delta_{m, s}+\frac{1}{2^{j+3}}, \\
b_{j, m, s}^{1,0}=0, & b_{j, m, s}^{1,1}=\frac{3}{4} \delta_{m, s}-\frac{1}{2^{j+3}} .
\end{array}
$$

Proof. Again, taking inner products with dual functions is the essential step. We have

$$
\begin{aligned}
a_{j, m, s}^{1,0}=\left\langle\tilde{\phi}_{j, s}^{0}, \phi_{j+1,2 m}^{1}\right\rangle \\
=-\frac{1}{2^{2 j+3}}\left(1-\delta_{s, m}\right) \cot \left(x_{j+1, m-s}\right)-\left\langle 1, \phi_{j+1,2 m}^{1}\right\rangle \\
\quad-2^{j+1} \sum_{\ell=0, \ell \neq s}^{2^{j+1}-1} \cot \left(x_{j+1, s-\ell}\right)\left(-\frac{1}{2^{4 j+6}}+\frac{1}{2^{3 j+4}} \delta_{\ell, m}\right)=0,
\end{aligned}
$$

while

$$
\begin{aligned}
\tilde{a}_{j, m, s}^{1,1}= & \left\langle\tilde{\phi}_{j, s}^{1}, \phi_{j+1,2 m}^{1}\right\rangle \\
= & -\frac{1}{2^{2 j+4}} \sum_{\ell=0, \ell \neq s, \ell \neq m}^{2^{j+1}-1} \cot \left(x_{j+1, s-\ell}\right) \cot \left(x_{j+1, m-\ell}\right) \\
& +2^{j+1} \sum_{\ell=0, \ell \neq s}^{2^{j+1}-1} \sin ^{-2}\left(x_{j+1, s-\ell}\right)\left(-\frac{1}{2^{4 j+6}}+\frac{1}{2^{3 j+4}} \delta_{\ell, m}\right) \\
& +2^{4 j+4} \frac{1}{3}\left(\frac{1}{2^{j}}+\frac{1}{2^{3 j+3}}\right)\left(-\frac{1}{2^{4 j+6}}+\frac{1}{2^{3 j+4}} \delta_{s, m}\right) \\
& +\sum_{\ell=0}^{2^{j+1}-1} 2^{2 j+2}\left(-\frac{1}{2^{4 j+6}}+\frac{1}{2^{3 j+4}} \delta_{\ell, m}\right) \\
= & -\frac{1}{2^{2 j+4}} \sum_{\ell=0, \ell \neq s, \ell \neq m}^{2^{j+1}-1} \cot \left(x_{j+1, s-\ell}\right) \cot \left(x_{j+1, m-\ell}\right) \\
& -\frac{1}{2^{3 j+5}} \sum_{\ell=0, \ell \neq s}^{2^{j+1}-1} \sin ^{-2}\left(x_{j+1, s-\ell}\right)+\frac{1}{2^{2 j+3}}\left(1-\delta_{s, m}\right) \sin ^{-2}\left(x_{j+1, s-m}\right) \\
& -\frac{1}{3}\left(\frac{1}{2^{j+2}}+\frac{1}{2^{3 j+5}}\right)+\frac{1}{3}\left(1+\frac{1}{2^{2 j+3}}\right) \delta_{s, m}+\frac{1}{2^{j+3}} .
\end{aligned}
$$

Replacing the cotangent sum as in the proof of Lemma 5.3, using the formulae (5.9) and (5.11) from Corollary 5.1 and a straightforward computation conclude this step. The computation of the wavelet coefficients follows as in Theorems 6.1 and 6.2 . 
Theorem 6.4. For any $j \in \mathbb{N}_{0}$ and $m=0, \ldots, 2^{j+1}-1$, we have

$$
\phi_{j+1,2 m+1}^{1}=\sum_{s=0}^{2^{j+1}-1}\left(\tilde{a}_{j, m, s}^{1,0} \phi_{j, s}^{0}+\tilde{a}_{j, m, s}^{1,1} \phi_{j, s}^{1}+\tilde{b}_{j, m, s}^{1,0} \psi_{j, s}^{0}+\tilde{b}_{j, m, s}^{1,1} \psi_{j, s}^{1}\right),
$$

where for $s=0, \ldots, 2^{j+1}-1$ the decomposition coefficients are given by

$$
\begin{aligned}
& \tilde{a}_{j, m, s}^{1,0}=-\frac{1}{2^{2 j+3}} \cot \left(x_{j+2,2 m+1-2 s}\right), \\
& \tilde{a}_{j, m, s}^{1,1}=-\frac{1}{2^{j+3}}-\frac{1}{2^{2 j+4}} \sin ^{-2}\left(x_{j+2,2 m+1-2 s}\right), \\
& \tilde{b}_{j, m, s}^{1,0}=\frac{1}{2^{2 j+3}} \cot \left(x_{j+2,2 m+1-2 s}\right), \\
& \tilde{b}_{j, m, s}^{1,1}=\frac{1}{2^{j+3}}+\frac{1}{2^{2 j+4}} \sin ^{-2}\left(x_{j+2,2 m+1-2 s}\right) .
\end{aligned}
$$

Proof. Finally, one obtains

$$
\begin{aligned}
\tilde{a}_{j, m, s}^{1,0} & =\left\langle\tilde{\phi}_{j, s}^{0}, \phi_{j+1,2 m}^{1}\right\rangle \\
& =-\frac{1}{2^{2 j+3}} \cot \left(x_{j+2,2 m+1-2 s}\right)-\left\langle 1, \phi_{j+1,2 m}^{1}\right\rangle+\frac{1}{2^{3 j+4}} \sum_{\ell=0, \ell \neq s}^{2^{j+1}-1} \cot \left(x_{j+1, s-\ell}\right),
\end{aligned}
$$

while

$$
\begin{aligned}
\tilde{a}_{j, m, s}^{1,1}= & \left\langle\tilde{\phi}_{j, s}^{1}, \phi_{j+1,2 m}^{1}\right\rangle \\
= & -\frac{1}{2^{2 j+4}} \sum_{\ell=0, \ell \neq s}^{2^{j+1}-1} \cot \left(x_{j+1, s-\ell}\right) \cot \left(x_{j+2,2 m+1-2 \ell}\right) \\
& -\frac{1}{2^{3 j+5}} \sum_{\ell=0, \ell \neq s}^{2^{j+1}-1} \sin ^{-2}\left(x_{j+1, s-\ell}\right)-\frac{1}{2^{j+3}}-\frac{1}{3}\left(\frac{1}{2^{j+2}}+\frac{1}{2^{3 j+5}}\right) .
\end{aligned}
$$

A final application of the cotangent formula (5.13) as well as (5.9), (5.10) and (5.11) yields

$$
\begin{aligned}
\tilde{a}_{j, m, s}^{1,1}= & \frac{1}{2^{2 j+4}} \sum_{\ell=0, \ell \neq s}^{2^{j+1}-1}\left[\cot \left(x_{j+2,2 m+1-2 s}\right)\left(\cot \left(x_{j+1, \ell-s}\right)+\cot \left(x_{j+2,2 m+1-2 \ell}\right)\right)+1\right] \\
& -\frac{1}{2^{3 j+5}} \frac{1}{3}\left(2^{2 j+2}-1\right)-\frac{1}{2^{j+3}}-\frac{1}{3}\left(\frac{1}{2^{j+2}}+\frac{1}{2^{3 j+5}}\right) .
\end{aligned}
$$

The wavelet coefficients are obtained by interpolation as in the previous theorems. $\square$

Using the notation and definitions introduced in $\S 4$, we can now completely describe the 16 circulant submatrices that constitute the decomposition matrix $D_{j}$. Indeed, $D_{j}$ has the structure

$$
\left(\begin{array}{cccc}
3 \cdot 2^{-2} I_{j}-2^{-j-3} E_{j} & 2^{-2} \tilde{T}_{j} & 2^{-2} I_{j}+2^{-j-3} E_{j} & -2^{-2} \tilde{T}_{j} \\
2^{-j-3} E_{j}+2^{-2 j-4} S_{j}^{T} & 2^{-2} T_{j}^{T}+2^{-2 j-4} U_{j}^{T} & -2^{-j-3} E_{j}-2^{-2 j-4} S_{j}^{T} & -2^{-2} T_{j}^{T}-2^{-2 j-4} U_{j}^{T} \\
0 & 2^{-2} I_{j}+2^{-j-3} E_{j} & 0 & 3 \cdot 2^{-2} I_{j}-2^{-j-3} E_{j} \\
-2^{-2 j-3} T_{j}^{T} & -2^{-j-3} E_{j}-2^{-2 j-4} S_{j}^{T} & 2^{-2 j-3} T_{j}^{T} & 2^{-j-3} E_{j}+2^{-2 j-4} S_{j}^{T}
\end{array}\right)
$$


where the submatrices are defined in $\S 4$, except for $\tilde{T}_{j}=\left(\cot \left(x_{j+1, s-n}\right)\right)_{n, s}$.

As $V_{j+1}=V_{j} \oplus W_{j}$, a function $f_{j+1} \in V_{j+1}$ can be written uniquely as

$$
f_{j+1}=f_{j}+g_{j}, \quad \text { with } \quad f_{j} \in V_{j} \quad \text { and } \quad g_{j} \in W_{j} .
$$

Using the basis functions of these spaces, one obtains

$$
\begin{gathered}
f_{j+1}=\sum_{s=0}^{2^{j+2}-1}\left(c_{s}^{0, j+1} \phi_{j+1, s}^{0}+c_{s}^{1, j+1} \phi_{j+1, s}^{1}\right), \\
f_{j}=\sum_{s=0}^{2^{j+1}-1}\left(c_{s}^{0, j} \phi_{j, s}^{0}+c_{s}^{1, j} \phi_{j, s}^{1}\right), \text { and } g_{j}=\sum_{s=0}^{2^{j+1}-1}\left(d_{s}^{0, j} \psi_{j, s}^{0}+d_{s}^{1, j} \psi_{j, s}^{1}\right) .
\end{gathered}
$$

Using coefficient vectors $\mathbf{c}_{j}^{i}=\left(c_{0}^{i, j}, c_{1}^{i, j}, \ldots, c_{2^{j+1}-1}^{i, j}\right)^{T}$ for $i=0,1$, respectively, as well as $\mathbf{d}_{j}^{i}=\left(d_{0}^{i, j}, d_{1}^{i, j}, \ldots, d_{2^{j+1}-1}^{i, j}\right)^{T}$, yields the representations

$f_{j+1}=\mathbf{c}_{j+1}^{0}{ }^{T} \Phi_{j+1}^{0}+\mathbf{c}_{j+1}^{1}{ }^{T} \Phi_{j+1}^{1}, f_{j}=\mathbf{c}_{j}^{0^{T}} \Phi_{j}^{0}+\mathbf{c}_{j}^{1 T} \Phi_{j}^{1}$, and $g_{j}=\mathbf{d}_{j}^{0^{T}} \Psi_{j}^{0}+\mathbf{d}_{j}^{1^{T}} \Psi_{j}^{1}$.

Since $\mathbf{c}_{j+1}{ }^{T} \Phi_{j+1}=\left(P_{j} \mathbf{c}_{j+1}\right)^{T} P_{j} \Phi_{j+1}$, the matrix form of the decomposition relation yields

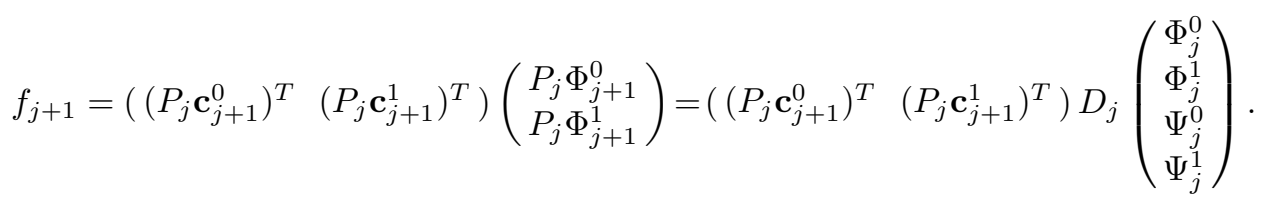

On the other hand, we have

$$
f_{j}+g_{j}=\left(\begin{array}{llll}
\mathbf{c}_{j}^{0^{T}} & \mathbf{c}_{j}^{1} & \mathbf{d}_{j}^{0^{T}} & \mathbf{d}_{j}^{1{ }^{T}}
\end{array}\right)\left(\begin{array}{c}
\Phi_{j}^{0} \\
\Phi_{j}^{1} \\
\Psi_{j}^{0} \\
\Psi_{j}^{1}
\end{array}\right) .
$$

Comparing coefficients and taking the transpose, we finally arrive at the matrix form of one step of the decomposition algorithm,

$$
\left(\begin{array}{c}
\mathbf{c}_{j}^{0} \\
\mathbf{c}_{j}^{1} \\
\mathbf{d}_{j}^{0} \\
\mathbf{d}_{j}^{1}
\end{array}\right)=D_{j}^{T}\left(\begin{array}{c}
P_{j} \mathbf{c}_{j+1}^{0} \\
P_{j} \mathbf{c}_{j+1}^{1}
\end{array}\right) .
$$

Multiplying by the inverse $\left(D_{j}^{T}\right)^{-1}=C_{j}^{T}$ yields the matrix representation of one step of the reconstruction algorithm,

$$
\left(\begin{array}{l}
P_{j} \mathbf{c}_{j+1}^{0} \\
P_{j} \mathbf{c}_{j+1}^{1}
\end{array}\right)=C_{j}^{T}\left(\begin{array}{c}
\mathbf{c}_{j}^{0} \\
\mathbf{c}_{j}^{1} \\
\mathbf{d}_{j}^{0} \\
\mathbf{d}_{j}^{1}
\end{array}\right)
$$


Altogether we obtain the following algorithms:

Algorithm 1 (Decomposition).

Input data: Function values and derivative values for some predetermined level $\eta$, $f\left(\frac{k \pi}{2^{\eta}}\right)=\left(L_{\eta} f\right)\left(\frac{k \pi}{2^{\eta}}\right)$ and $f^{\prime}\left(\frac{k \pi}{2^{\eta}}\right)=\left(L_{\eta} f\right)^{\prime}\left(\frac{k \pi}{2^{\eta}}\right), \quad k=0, \ldots, 2^{\eta+1}-1$.

Step 1: Set

$$
\mathbf{c}_{\eta}^{0}=\left(f\left(\frac{k \pi}{2^{\eta}}\right)\right)_{k=0}^{2^{\eta+1}-1} \quad \text { and } \quad \mathbf{c}_{\eta}^{1}=\left(f^{\prime}\left(\frac{k \pi}{2^{\eta}}\right)\right)_{k=0}^{2^{\eta+1}-1} .
$$

Step 2: Repeat for $j=\eta-1, \ldots, 0$ the computation

$$
\left(\begin{array}{c}
\mathbf{c}_{j}^{0} \\
\mathbf{c}_{j}^{1} \\
\mathbf{d}_{j}^{0} \\
\mathbf{d}_{j}^{1}
\end{array}\right)=D_{j}^{T}\left(\begin{array}{c}
P_{j} \mathbf{c}_{j+1}^{0} \\
P_{j} \mathbf{c}_{j+1}^{1}
\end{array}\right) .
$$

Output data: The wavelet coefficients $\mathbf{d}_{j}^{0}$ and $\mathbf{d}_{j}^{1}$ for $j=0, \ldots, \eta-1$ and the lowest-level scaling function coefficients $\mathbf{c}_{0}^{0}$ and $\mathbf{c}_{0}^{1}$.

Algorithm 2 (Reconstruction).

Input data: The wavelet coefficients $\mathbf{d}_{j}^{0}$ and $\mathbf{d}_{j}^{1}$ for $j=0, \ldots, \eta-1$ and the lowest-level scaling function coefficients $\mathbf{c}_{0}^{0}$ and $\mathbf{c}_{0}^{1}$.

Step 1: Repeat for $j=0, \ldots, \eta-1$ the computation

$$
\left(\begin{array}{c}
P_{j} \mathbf{c}_{j+1}^{0} \\
P_{j} \mathbf{c}_{j+1}^{1}
\end{array}\right)=C_{j}^{T}\left(\begin{array}{c}
\mathbf{c}_{j}^{0} \\
\mathbf{c}_{j}^{1} \\
\mathbf{d}_{j}^{0} \\
\mathbf{d}_{j}^{1}
\end{array}\right) .
$$

Output data: The scaling function coefficients on level $\eta$, i.e., $\mathbf{c}_{\eta}^{0}$ and $\mathbf{c}_{\eta}^{1}$. For perfect reconstruction, these are the vectors

$$
\left(\left(L_{\eta} f\right)\left(\frac{k \pi}{2^{\eta}}\right)\right)_{k=0}^{2^{\eta+1}-1} \text { and }\left(\left(L_{\eta} f\right)^{\prime}\left(\frac{k \pi}{2^{\eta}}\right)\right)_{k=0}^{2^{\eta+1}-1} .
$$

Note that the efficiency of these two algorithms depends essentially on the proper implementation of the matrix/vector multiplications using Fast-Fourier-Transform techniques. As in [6, Chapter 3], the circulant submatrices of $C_{j}$ and $D_{j}$ can be factored into the product of some so-called Fourier matrices and the diagonal matrix of the eigenvalues, which can be computed directly. All the necessary computations for these factorizations only need to be done once for a predetermined number of levels. Thus, Algorithms 1 and 2 need $\mathcal{O}\left(j 2^{j}\right)$ operations, which is best possible for this type of matrix calculations, but this does not realize the best possible pyramid algorithms of order $\mathcal{O}\left(2^{j}\right)$ available for some other wavelet schemes. Thus, this fully computable trigonometric multiresolution analysis with explicit algebraic 
formulas yields "almost optimal" complexity. Another algorithmic advantage of the interpolatory approach is the simplicity of finding a suitable projection onto $V_{j}$, just by function and derivative evaluation in the dyadic nodes.

\section{Conclusion}

Figure 1 shows the two scaling functions $\phi_{4,16}^{0} \in V_{4}$ and $\phi_{4,16}^{1} \in V_{4}$ and Figure 2 the two wavelets $\psi_{4,16}^{0} \in W_{4}$ and $\psi_{4,16}^{1} \in W_{4}$. Observe their respective interpolatory properties for the knot sequence $x_{4, n}=\frac{n \pi}{16}$ for $n=0, \ldots, 31$. Recall also that the scaling functions and wavelets at higher levels are not just scaled versions of the ones at level zero, owing to their interpolatory properties.

Figures 3, 4, 5 and 6 illustrate the use of trigonometric wavelet decompositions to detect discontinuities in higher-order derivatives of a function. In this case, a cubic B-spline with equidistant knots at $\{1,2,3,4,5\}$, i.e.,

$$
f(x):=\left\{\begin{array}{lc}
0, & x \in[0,1], \\
\frac{1}{6}(x-1)^{3}, & x \in(1,2], \\
\frac{1}{6}\left(-3(x-1)^{3}+12(x-1)^{2}-12(x-1)+4,\right. & x \in(2,3], \\
\frac{1}{6}\left(-3(5-x)^{3}+12(5-x)^{2}-12(5-x)+4,\right. & x \in(3,4], \\
\frac{1}{6}(5-x)^{3}, & x \in(4,5], \\
0, & x \in(5,2 \pi]
\end{array}\right.
$$

(properly periodized to generate a $2 \pi$-periodic function), was interpolated by an element of $V_{10}$ (Figure 3 ) using the operator $L_{10}$ of Definition 2.3. The breakpoints of the spline, where its third derivative has jump discontinuities, can be clearly detected in both wavelet components, i.e., the one corresponding to the interpolation of function values (W0 in Figure 4) spanned by the functions $\psi_{9, n}^{0}$ and the one corresponding to the first derivative values (W1 in Figure 4) spanned by the functions $\psi_{9, n}^{1}$, created by one decomposition step and shown in Figure 4. The detection effect is more and more blurred in subsequent decomposition steps, as illustrated by the wavelet parts for the levels 8 and 7 , shown in Figures 5 and 6 . In a whole series of numerical tests it always happened that both wavelet components (for function values and derivative values) showed similar edge detection capabilities.

As an outlook on further ongoing research, recall from [11] that Lagrange interpolation for spaces of the type $\operatorname{span}\left\{T_{2^{j}-1}, \cos 2^{j} x\right\}$ is associated with wavelets that interpolate in the midpoints of the underlying node sequence. On the other hand, the Hermite approach of this paper for spaces of the type $\operatorname{span}\left\{T_{2^{j}-1}, \sin 2^{j} x\right\}$ gives rise to wavelets that interpolate fundamental data in the given nodes. The regularity of trigonometric Hermite-Birkhoff interpolation on equidistant nodes has been thoroughly investigated in [2]. For a proper choice of interpolation nodes, nested sequences of spaces spanned by Hermite-Birkhoff interpolants can be found. Consequently, this poses the question as to what kind of interpolatory behavior the wavelet functions spanning the corresponding relative orthogonal complements will possess. This problem is currently under investigation. 
$\Lambda$ 
716

EWALD QUAK
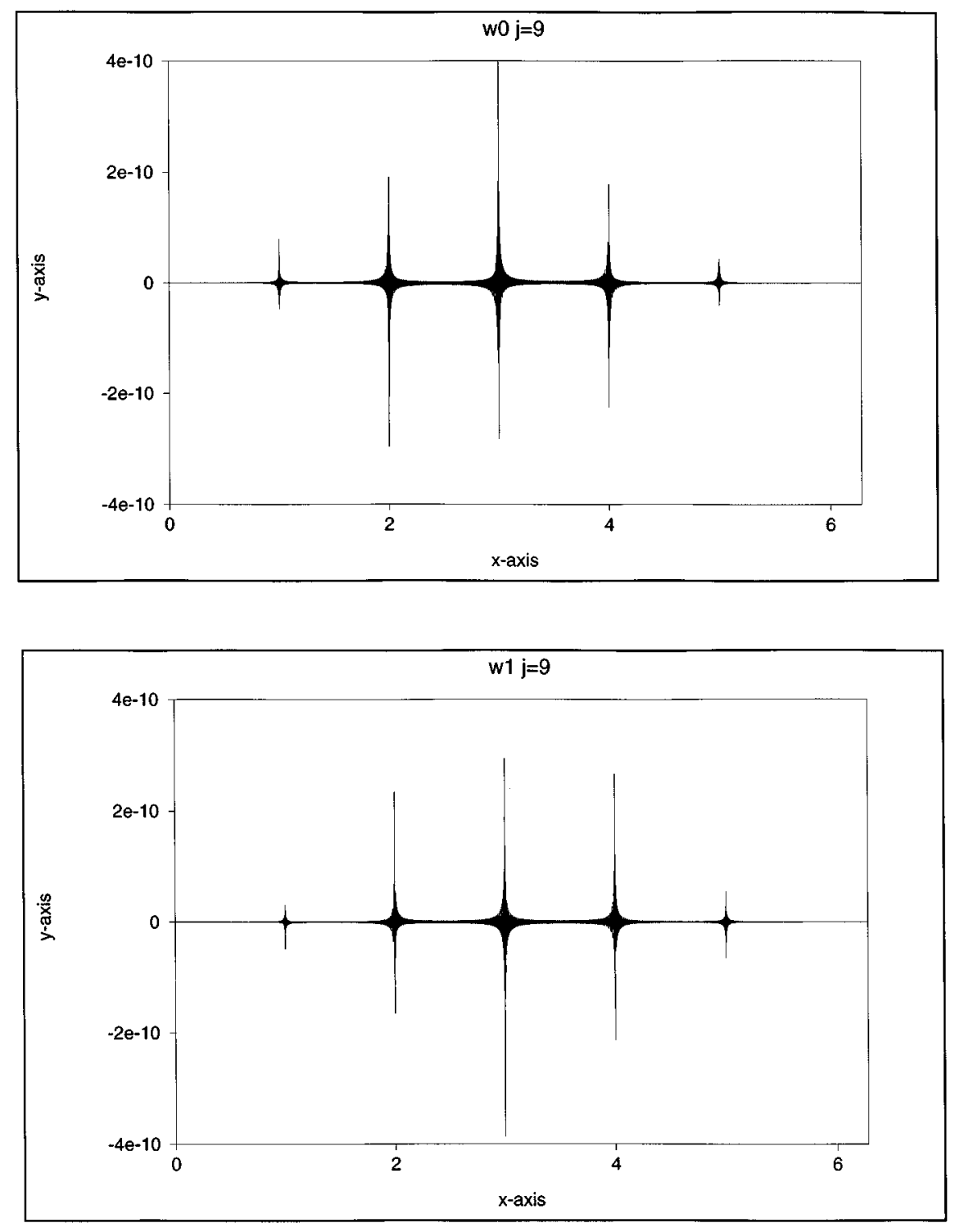

FiguRE 4 
TRIGONOMETRIC HERMITE WAVELETS
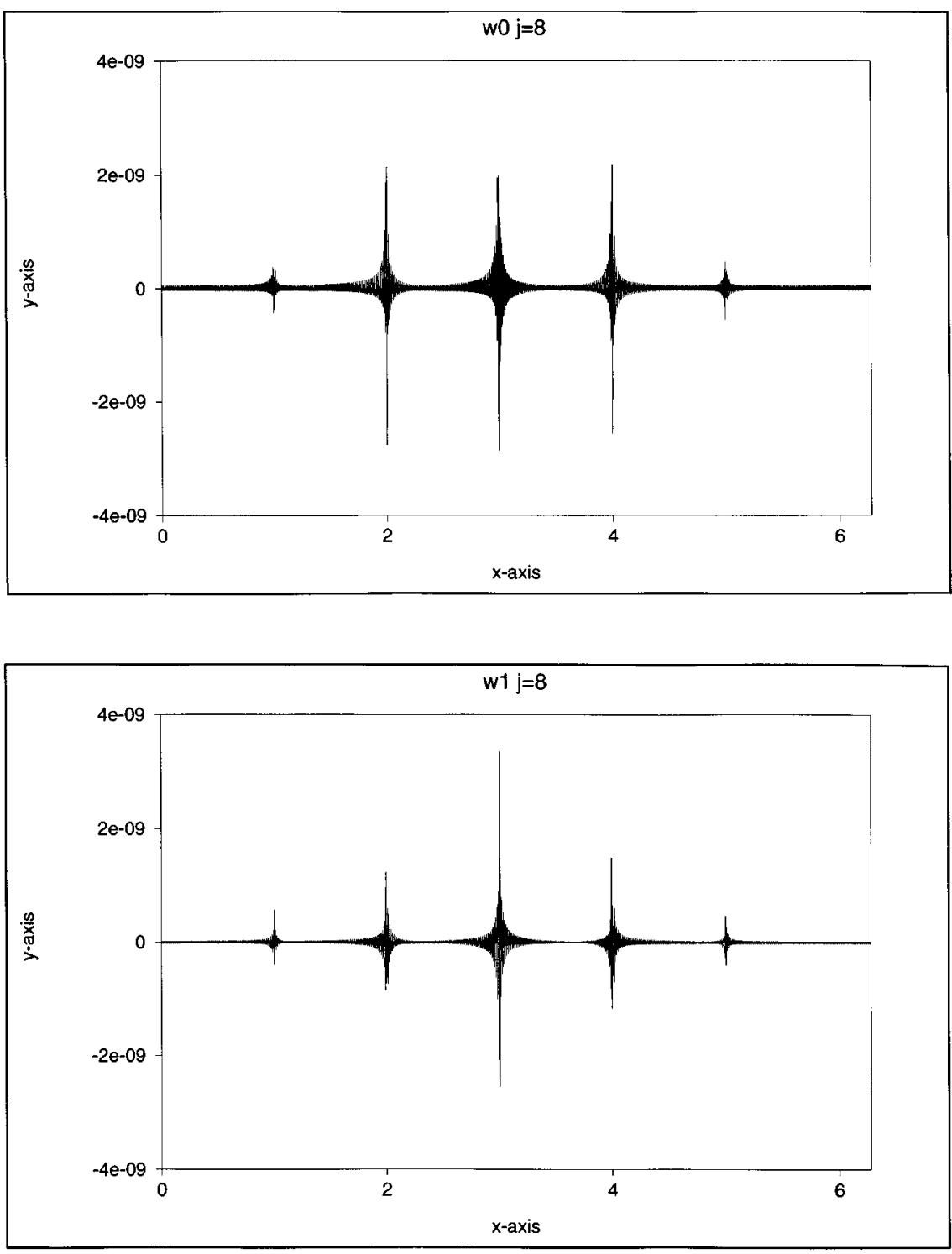

FIGURE 5 

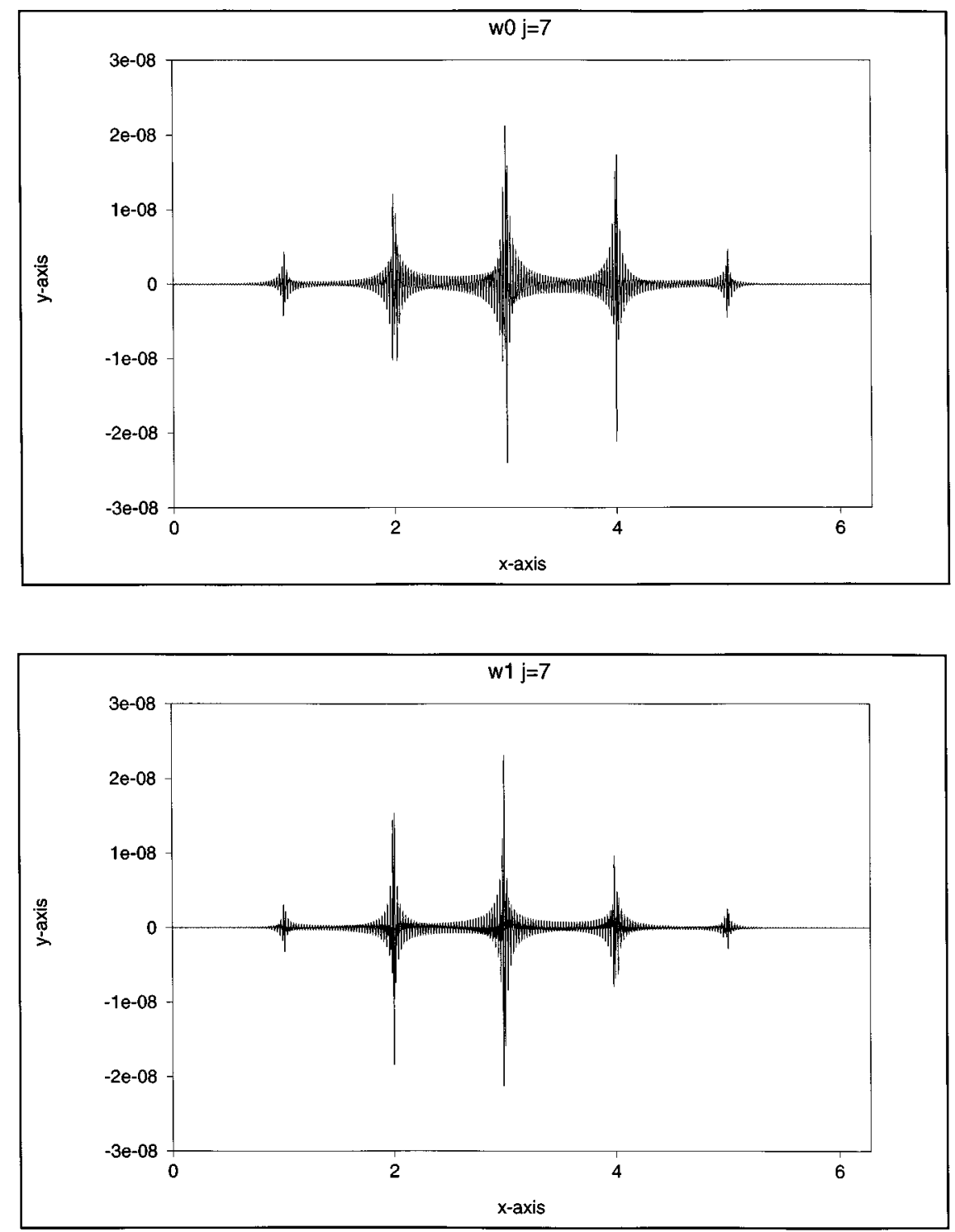

Figure 6

\section{APPENDIX}

In order to prove Theorem 2.3, a suitable inequality of Marcinkiewicz-Zygmund type is needed. Although it is possible to derive the following Theorem A.1 as a special case of the general investigations by $\mathrm{Y}$. Xu [16], a direct proof will be given for the sake of completeness and in order to illustrate the behavior of the constants involved, which cannot be easily obtained from the general result. 
For a trigonometric polynomial $T$, consider the following expressions, where $1 \leq p \leq \infty$ (with the usual supremum modification for $p=\infty$ ):

$$
\|T\|_{p}:=\left(\frac{1}{2 \pi} \int_{0}^{2 \pi}|T(x)|^{p} d x\right)^{1 / p}
$$

and

$$
\|T\|_{p, j}:=\left(\frac{1}{2^{j+1}} \sum_{k=0}^{2^{j+1}-1}\left|T\left(x_{j, k}\right)\right|^{p}\right)^{1 / p}
$$

where the $x_{j, k}$ 's are the equally spaced knots introduced in $\S 2$.

An inequality by Nikol'skii [15, Chapter 4.9] states that for $1 \leq p \leq \infty$ and any $T \in T_{n}$

$$
\|T\|_{p} \leq \sup _{x}\left(\frac{1}{N} \sum_{k=0}^{N-1}\left|T\left(x-\frac{2 k \pi}{N}\right)\right|^{p}\right)^{1 / p} \leq\left(1+\frac{2 n \pi}{N}\right)\|T\|_{p} .
$$

Theorem A.1 (Marcinkiewicz-Zygmund type inequality). For a trigonometric polynomial $T \in V_{j}$ and $1 \leq p \leq \infty$, it holds that

$$
\frac{1}{2(1+2 \pi)}\left(\|T\|_{p, j}+\frac{1}{2^{j+1}}\left\|T^{\prime}\right\|_{p, j}\right) \leq\|T\|_{p} \leq\|T\|_{p, j}+\frac{1}{2^{j+1}} C_{p, j}\left\|T^{\prime}\right\|_{p, j},
$$

with the constant $C_{p, j}$ depending only on $p$ for $1<p<\infty$, and $C_{p, j}=j C_{p}$, for a constant $C_{p}$ depending only on $p$, in the cases $p=1$ and $p=\infty$.

Proof. The full-length formulation of the statement of the theorem is

$$
\begin{aligned}
& \frac{1}{1+2 \pi}\left\{\left(\frac{1}{2^{j+1}} \sum_{k=0}^{2^{j+1}-1}\left|u_{k}\right|^{p}\right)^{1 / p}+\left(\frac{1}{2^{(j+1)(p+1)}} \sum_{k=0}^{2^{j+1}-1}\left|v_{k}\right|^{p}\right)^{1 / p}\right\} \\
& \leq\left\|\sum_{k=0}^{2^{j+1}-1}\left(u_{k} \phi_{j, k}^{0}+v_{k} \phi_{j, k}^{1}\right)\right\|_{p} \\
& \leq\left\{\left(\frac{1}{2^{j+1}} \sum_{k=0}^{2^{j+1}-1}\left|u_{k}\right|^{p}\right)^{1 / p}+C_{p, j}\left(\frac{1}{2^{(j+1)(p+1)}} \sum_{k=0}^{2^{j+1}-1}\left|v_{k}\right|^{p}\right)^{1 / p}\right\} .
\end{aligned}
$$

For

$$
T=\sum_{k=0}^{2^{j+1}-1}\left(u_{k} \phi_{j, k}^{0}+v_{k} \phi_{j, k}^{1}\right)
$$

inequality $(*)$ yields

$$
\left(\frac{1}{2^{j+1}} \sum_{k=0}^{2^{j+1}-1}\left|u_{k}\right|^{p}\right)^{1 / p} \leq\left(1+\frac{2\left(2^{j+1}-1\right) \pi}{2^{j+1}}\right)\|T\|_{p}
$$


as well as in combination with the classical Bernstein inequality

$$
\left(\frac{1}{2^{j+1}} \sum_{k=0}^{2^{j+1}-1}\left|v_{k}\right|^{p}\right)^{1 / p} \leq(1+2 \pi)\left\|T^{\prime}\right\|_{p} \leq(1+2 \pi) 2^{j+1}\|T\|_{p}
$$

i.e., the left inequality of the theorem.

For the other inequality, we split up as follows:

$$
\|T\|_{p} \leq\left\|\sum_{k=0}^{2^{j+1}-1} u_{k} \phi_{j, k}^{0}\right\|_{p}+\left\|\sum_{k=0}^{2^{j+1}-1} v_{k} \phi_{j, k}^{1}\right\|_{p} .
$$

By Hölder's inequality (with $1 / p+1 / q=1$ ), one obtains for the second term, if $1<q<\infty$,

$$
\begin{aligned}
\left\|\sum_{k=0}^{2^{j+1}-1} v_{k} \phi_{j, k}^{1}\right\|_{p} & \leq\left(\frac{1}{2 \pi} \int_{0}^{2 \pi}\left(\sum_{k=0}^{2^{j+1}-1}\left|v_{k}\right|^{p}\right)\left(\sum_{k=0}^{2^{j+1}-1}\left|\phi_{j, k}^{1}\right|^{q}\right)^{p / q} d x\right)^{1 / p} \\
& \leq\left(2^{j+1}\left\|T^{\prime}\right\|_{p, j}^{p} \frac{1}{2 \pi} \int_{0}^{2 \pi}\left(\sum_{k=0}^{2^{j+1}-1}\left|\phi_{j, k}^{1}\right|^{q}\right)^{p / q} d x\right)^{1 / p} \\
& =2^{j+1}\left\|T^{\prime}\right\|_{p, j}\left(\frac{1}{2 \pi} \int_{0}^{2 \pi}\left(\frac{1}{2^{j+1}} \sum_{k=0}^{2^{j+1}-1}\left|\phi_{j, k}^{1}\right|^{q}\right)^{p / q} d x\right)^{1 / p} \\
& \leq 2^{j+1}\left\|T^{\prime}\right\|_{p, j}\left(\frac{1}{2 \pi} \int_{0}^{2 \pi}\left((1+2 \pi)\left\|\phi_{j, 0}^{1}\right\|_{q}\right)^{p} d x\right)^{1 / p} \\
& <2^{j+1}\left\|T^{\prime}\right\|_{p, j}(1+2 \pi)\left\|\phi_{j, 0}^{1}\right\|_{q},
\end{aligned}
$$

where $(*)$ was used again.

Similarly, one can also use $(*)$ to produce the analogous final estimates for $q=1$ and $q=\infty$. The norm of $\phi_{j, 0}^{1}$ now behaves as stated, i.e., it is a constant depending on $p$ for $1<p<\infty$, while for $p=1$ and $p=\infty$ an additional logarithmic term appears (note that $\log 2^{j}=j$ ), see for example [17, Vol. I, Ch. 2.12 and 13].

It remains to investigate the behavior of the first term. Again by Zygmund [17, Vol. II, Ch. 10], there exists a function $g \in L_{2 \pi}^{q}$ with $\|g\|_{q}=1$ such that

$$
\left\|\sum_{k=0}^{2^{j+1}-1} u_{k} \phi_{j, k}^{0}\right\|_{p}=\frac{1}{2 \pi} \int_{0}^{2 \pi} \sum_{k=0}^{2^{j+1}-1} u_{k} \phi_{j, k}^{0}(t) g(t) d t
$$

and with Hölder's inequality

$$
\leq\|T\|_{p, j}\left(\frac{1}{2^{j+1}} \sum_{k=0}^{2^{j+1}-1}\left|\frac{1}{2 \pi} \int_{0}^{2 \pi} 2^{j+1} \phi_{j, k}^{0}(t) g(t) d t\right|^{q}\right)^{1 / q} .
$$


Jensen's inequality yields

$$
\begin{aligned}
& \left(\frac{1}{2^{j+1}} \sum_{k=0}^{2^{j+1}-1}\left|\frac{1}{2 \pi} \int_{0}^{2 \pi} 2^{j+1} \phi_{j, k}^{0}(t) g(t) d t\right|^{q}\right)^{1 / q} \\
& \leq\left(\frac{1}{2^{j+1}} \sum_{k=0}^{2^{j+1}-1} \frac{1}{2 \pi} \int_{0}^{2 \pi} 2^{j+1} \phi_{j, k}^{0}(t)|g(t)|^{q} d t\right)^{1 / q}=\|g\|_{q}(=1),
\end{aligned}
$$

using the positivity of $\phi_{j, k}^{0}(t)$ and the fact that $\sum_{k=0}^{2^{j+1}-1} \phi_{j, k}^{0}(t)=1$, as $L_{j}$ reproduces constants, thus completing the proof of Theorem A.1.

Applying Theorem A.1, one can now prove Theorem 2.3, following exactly the steps as given in the general situation by $\mathrm{Xu}[16, \S 3]$.

\section{ACKNOWLEDGEMENTS}

The author thanks the referee for very helpful suggestions and Dr. Jürgen Prestin for several discussions, which led to an improvement of the paper.

\section{REFERENCES}

1. P. Auscher, Wavelets with boundary conditions on the interval, Wavelets: a Tutorial in Theory and Applications (C.K. Chui, ed.), Academic Press, Boston, 1992, pp. 237-256. MR 93c:42029

2. A. S. Cavaretta, A. Sharma and R. S. Varga, Lacunary trigonometric interpolation on equidistant nodes, Quantitative Approximation (R. A. DeVore and K. Scherer, eds.), Academic Press, New York, 1980, pp. 63-80. MR 81k:42004

3. C. K. Chui, An Introduction to Wavelets, Academic Press, Boston, 1992. MR 93f:42055

4. C. K. Chui and H. N. Mhaskar, On trigonometric wavelets, Constr. Approx. 9 (1993), 167-190. MR 94c: 42002

5. C. K. Chui and J. Z. Wang, On compactly supported spline wavelets and a duality principle, Trans. Amer. Math. Soc. 330 (1992), 903-915. MR 92f:41020

6. P. J. Davis, Circulant matrices, Wiley Interscience, New York, 1979. MR 81a:15003

7. T. N. T. Goodman, Interpolatory Hermite spline wavelets, J. Approx. Theory 78 (1994), 174-189. CMP 94:15

8. Y. W. Koh, S. L. Lee and H. H. Tan, Periodic orthogonal splines and wavelets, Applied and Computational Harmonic Analysis 2 (1995), 201-218. CMP 95:15

9. R. A. Lorentz and A. A. Sahakian, Orthogonal trigonometric Schauder bases of optimal degree for $C(K)$, Journal of Fourier Analysis and Applications 1 (1994), 103-112. CMP 95:05

10. D. Offin and K. Oskolkov, A note on orthonormal polynomial bases and wavelets, Constr. Approx. 9 (1993), 319-325. MR 94f:42047

11. J. Prestin and E. Quak, Trigonometric interpolation and wavelet decompositions, Numerical Algorithms 9 (1995), 293-317. CMP 95:15

12. _ A duality principle for trigonometric wavelets, Wavelets, Images, and Surface Fitting (P. J. Laurent, A. Le Méhauté and L. L. Schumaker, eds.), A K Peters, Boston, 1994, pp. 407418. CMP 95:03

13. _ Decay properties of trigonometric wavelets, Proceedings of the Cornelius Lanczos International Centenary Conference (J. D. Brown, M. T. Chu, D. C. Ellison and R. J. Plemmons, eds.), SIAM, Philadelphia, 1994, pp. 413-415.

14. A. A. Privalov, On an orthogonal trigonometric basis, Mat. Sbornik 182 (3) (1991), 384-394. MR 92f: 42005

15. A. F. Timan, Theory of approximation of functions of a real variable, Pergamon Press, Oxford, 1963. MR 33:465 
EWALD QUAK

16. Y. Xu, The generalized Marcinkiewicz-Zygmund inequality for trigonometric polynomials, J. Math. Anal. Appl. 161 (1991), 447-456. MR 93f:42008

17. A. Zygmund, Trigonometric Series, Cambridge University Press, Cambridge, 1959. MR 21:6498

Center for Approximation Theory, Department of Mathematics, Texas A\&M UniverSity, College Station, Texas 77843-3368

E-mail address: quak@math.tamu.edu 